
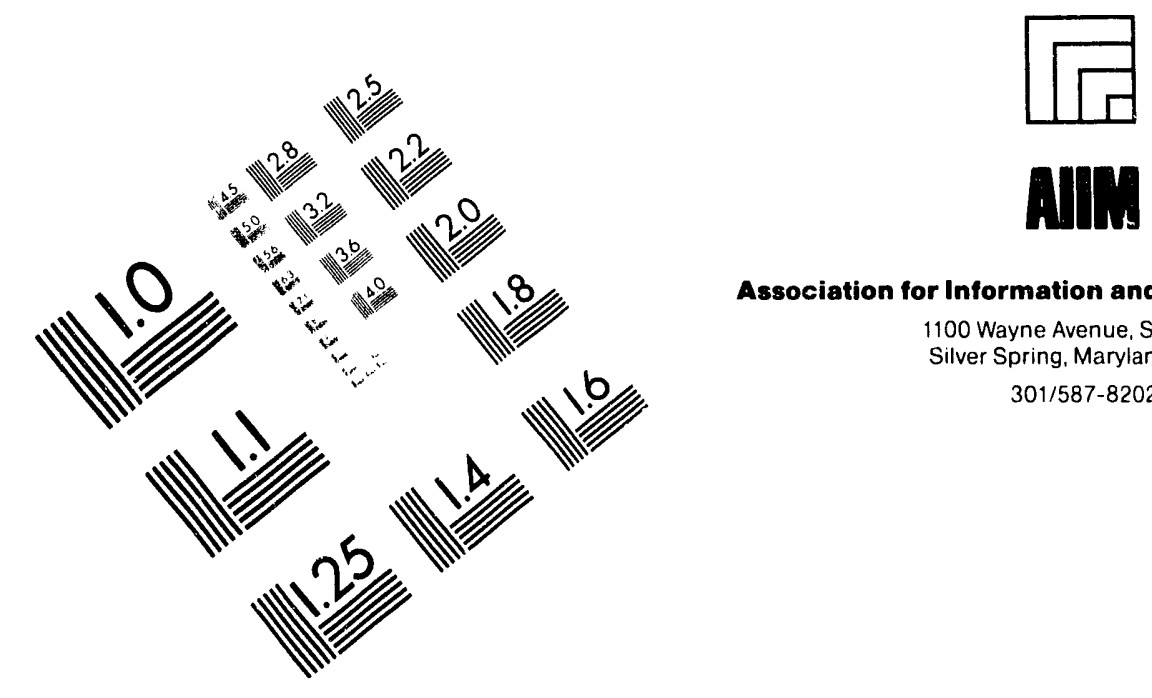

Association for Information and Image Management

1100 Wayne Avenue. Suite 1100

Silver Spring, Maryland 20910

301/587-8202

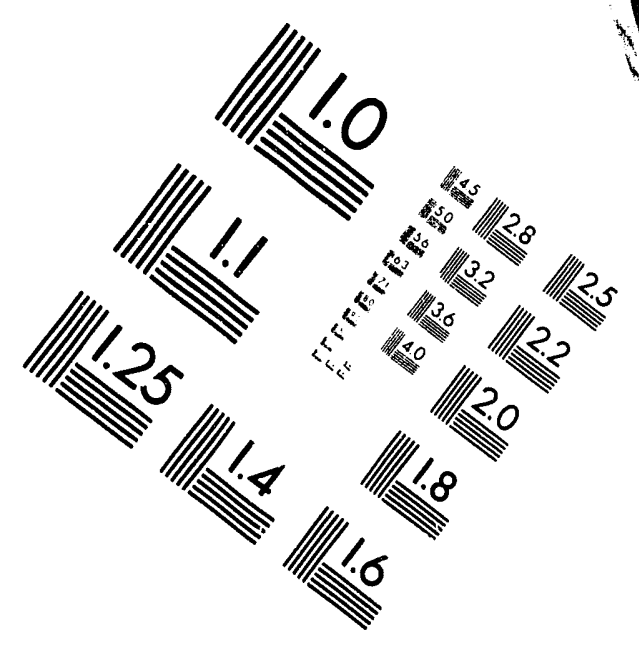

\title{
Centimeter
}

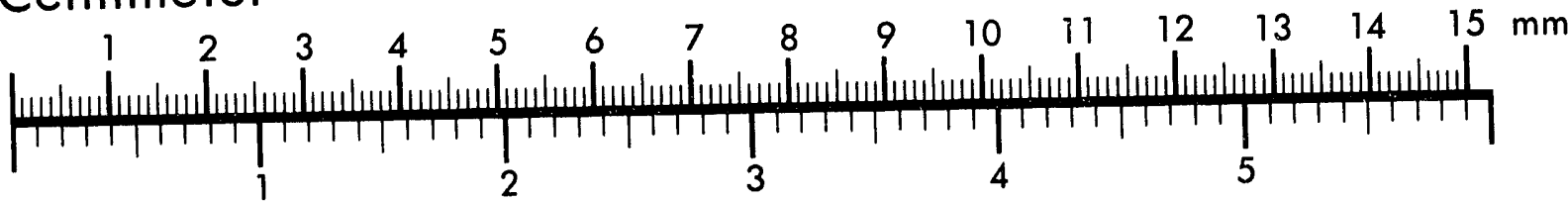
Inches
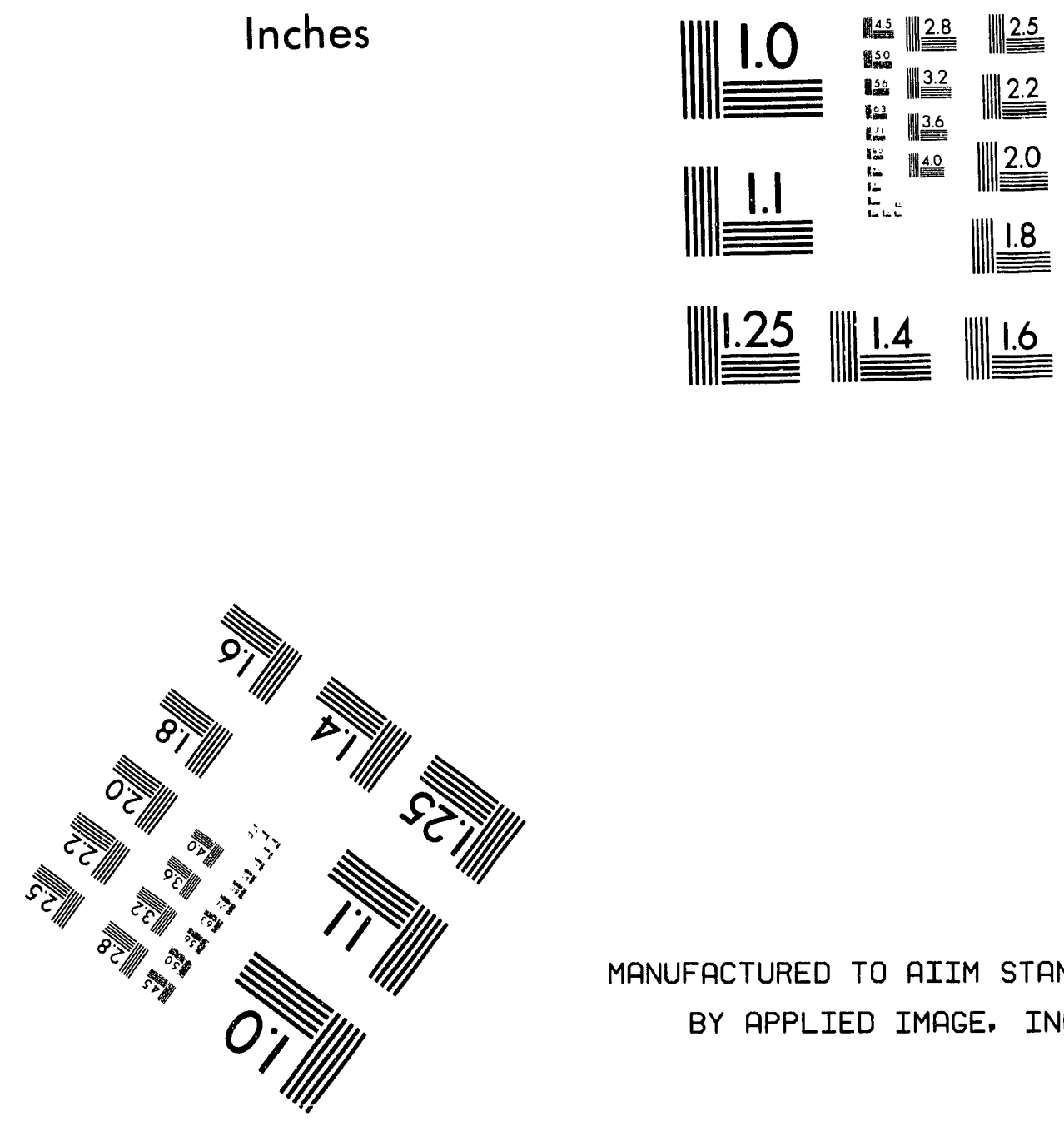

MANUFACTURED TO AIIM STANDARDS

BY APPLIED IMAGE, INC.

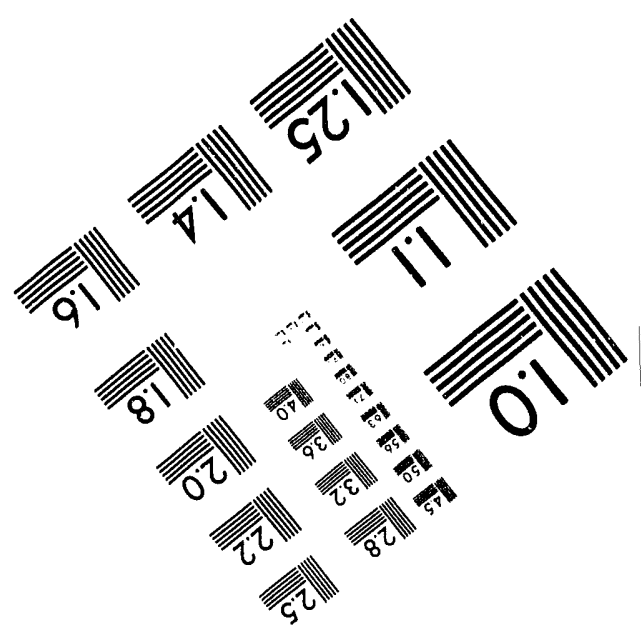



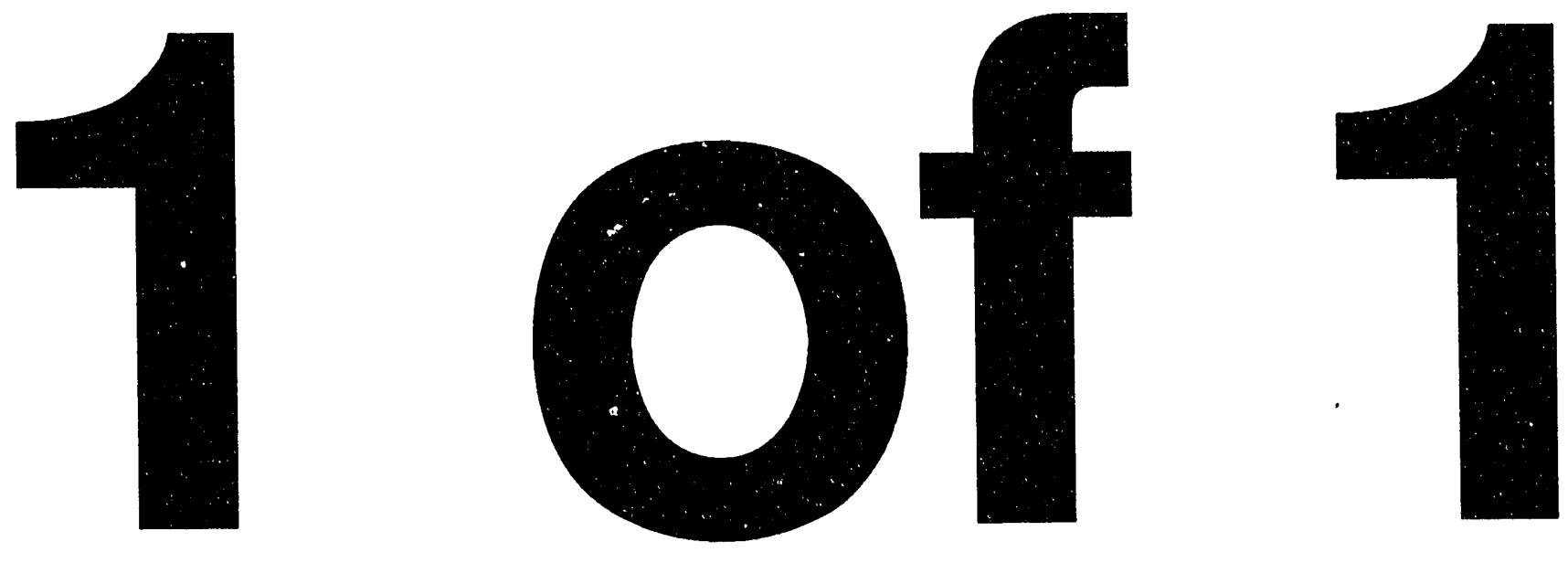
UC- $420,421,426,427$

\author{
EVAI_UATION OF POTENTIAL RUNAWAY GENERATION \\ IN LARGE-TOKAMAK DISRUPTIONS
}

\title{
BY
}

H.H. FLEISCHMANN AND S.J. ZWEBEN

JUNE, 1993

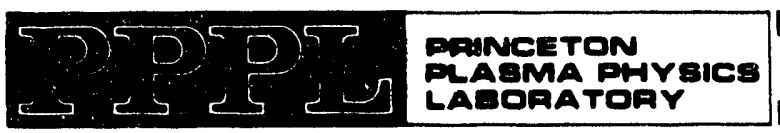

\section{masma PHYBice} LABOMATOAY

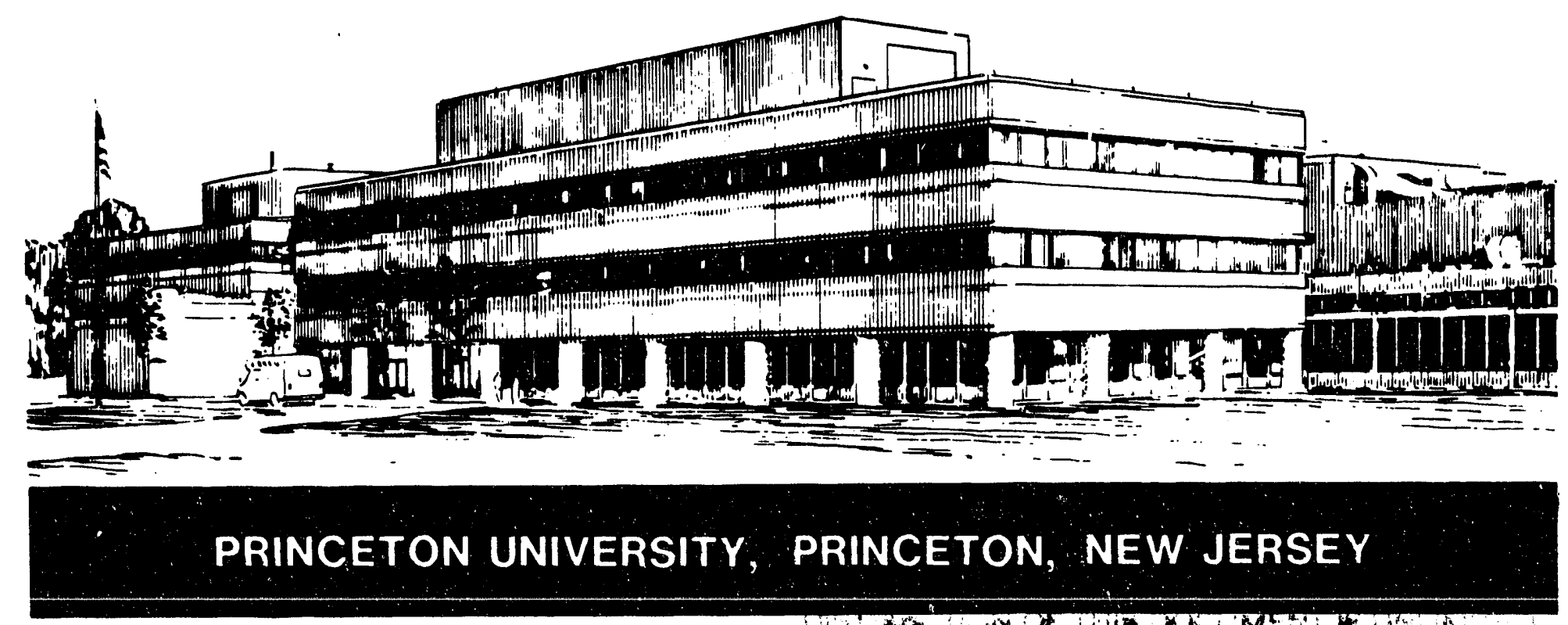




\section{NOTICE}

This report was prepared as an account of work sponsored by an agency of the United States Government. Neither the United States Government nor any agency thereof, nor any of their employees, makes any warranty, express or implied, or assumes any legal liability or responsibility for the accuracy, completeness, or usefulness of any information, apparatus, product, or process disclosed, or represents that its use would not infringe privately owned rights. Reference herein to any specific commercial produce, process, or service by trade name, trademark, manufacturer, or otherwise, does not necessarily constitute or imply its endorsement, recommendation, or favoring by the United States Government or any agency thereof. The views and opinions of authors expressed herein do not necessarily state or reflect those of the United States Government or any agency thereof.

\section{NOTICE}

This report has been reproduced from the best available copy.

Available in paper copy and microfiche.

Number of pages in this report: 52

DOE and DOE contractors can obtain copies of this report from:

Office of Scientific and Technical Information

P.O. Box 62

Oak Ridge, TN 37831;

(615) $576-8401$.

This report is publicly available from the:

National Technical Information Service

Department of Commerce

5285 Port Royal Road

Springfield, Virginia 22161

(703) $487-4650$ 


\title{
Evaluation of Potential Runaway Generation in Large-Tokamak Disruptions
}

\author{
H. H. Fleischmann, and S. J. Zweben \\ Plasma Physics Laboratory, Princeton University, Princeton, N. J. 08543 \\ and \\ School of Applied and Engineering Physics, Cornell University, Ithaca, N.Y. 14853
}

Abstract

A detailed evaluation of various potential mechanisms for the generation of strong runaway beams during disruptions of largetokamak devices, including TFTR, JET, DIIID and ITER, is performed based on typical operating parameters of these devices and the presently accepted disruption model. The main results include: (i) In the existing devices, the evaporative "Dreicer" process by itself can lead to sizable runaway beams in disruptions of high-currentmedium-to-low- $n_{e}$ discharges. In ITER, such runaways are expected mainly for discharges with $n_{e}$ values sizably smaller than the projected typical ones. (ii) Runaway generation also may occur during post-thermal-quench period through the untrapping of trapped hot-thermal electrons remaining from the pre-thermal-quench plasma; this process may be directly important in particular in disruptions of high- $T_{e}$ discharges with details depending on the time required for re-closure of the magnetic surfaces. Both processes, (i) and (ii), will occur and be completed mostly during the initial few $100 \mu \mathrm{sec}$ after the thermal quench. (iii) Subsequently, close collisions of runaways with cold plasma electrons generally will lead to an exponential growth ("avalanching") of runaway populations generated by processes (i) and/or (ii) and/or others; this process will be effective in particular during the current quench phase and 
will continue until the resulting runaway beam will carry essentially all of the remaining discharge current. In presently existing devices, possible avalanche factors of up to $10^{2}-10^{5}$ may be expected; in ITER, avalanche factors of up to $10^{10}-10^{15}$ - if not properly suppressed - are expected to lead to strong runaway beams in most disruptions, except those at particularly high densities. At the same time, avalanching will shift the main part of their energy spectrum down to relatively low energies around 10-20 MeV, and may sizably change the spatial distribution of the runaways. The behavior and decay of the generated runaway beams is to be investigated in a separate study. 


\section{List of Symbols Used}

E = B-paralel electric field

$E_{D} \quad=$ Dreicer normalization field

$f_{e e}=n_{e} e^{4} \ln \Lambda /\left[4 \pi \varepsilon_{o}{ }^{2}\left(k T_{e}\right)^{3 / 2} m_{e}{ }^{1 / 2}\right]$

$=$ electron-electron collision frequency

$f_{e o}=Z_{\text {eff }} f_{e e}=$ electron-ioon collsiion frequency

$F(\tau)=$ normalized decay function of trapped hot electrons

go $=\left(\varepsilon_{i}-\varepsilon_{C}\right) / \varepsilon_{i}=j_{r a, o} / j_{0}=$ fraction of total current carried by runaways when Dreicer process stops (since $\varepsilon$ falls below $\varepsilon_{\mathrm{C}}$ )

$\mathrm{G} \quad=\left(d n_{r a} / d t\right)=$ density rate for runaway generation

$G_{D} \quad=\left(d n_{r a} / d t\right)_{D}=$ rate density for Dreicer-like runaway generation

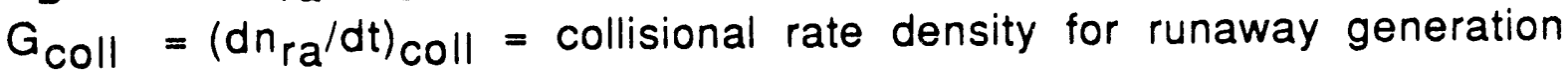

$I_{\text {tot }}=$ total discharge current

$\delta l_{\text {tot }}=$ change of total discharge current

$<j>\quad=I_{\text {tot }} / \pi a b=$ average discharge current

$j_{C r}=\varepsilon_{C}\left(E_{D} / \rho\right)=$ critical current density below which Dreicer process effectively stops

jo $\quad=\left(j_{q}<j>\right)^{1 / 2}=$ "typical" initial current density, pertaining to pre-disruption plasma, generally used as basis for estimates

$i_{p} \quad=$ current density carried by thermal plasma

$\mathrm{j}_{\mathrm{q}}=$ current density for $\mathrm{q}(0)=1$ on the magnetic axis

Ir a = current density carried by runaways

$j_{r a, 0}=j_{0}-j_{c r}=$ runaway current when Dreicer generation of runaways effectively stops

$j_{\text {to }}=j_{r a}+j_{p}=$ total current density

$n_{e} \quad=$ plasma density

$n_{\text {ra }}=$ density of runaways

$P_{C}=\left(2 m_{\theta} W_{C}\right)^{1 / 2}=$ electron momentum at critical Dreicer energy $W_{C}$

$P_{\text {ra }}=$ momentum of runaway

$p_{r a, 0}=j_{0} / e c n_{\theta}=$ percentage of relativistic runaways required for carrying full initial current density, jo

$P_{\text {trap }}=$ percentage of trapped hot electrons 
$p_{\text {trap,o }}=$ percentage of trapped electrons just after thermal quench

$R_{m}=$ mirror ratio on field surface

$t^{\prime} \quad=$ time after thermal quench

$t_{a c c}=m_{e} c / e E=$ time required for runaways to reach relativistic speed

$t_{f}=$ time of reclosure of magnetic field lines after thermal quench

$t_{r a, j o}=$ time at which $n_{r a}$ reaches the level $n_{e} g_{o} p_{r a, 0}$, at which the runaways would stop further runaway generation via the Dreicer process if they are fully relativistic

$\mathrm{t}_{\mathrm{qu}}=$ cooling time in thermal quench (generally $\sim$ few $100 \mu \mathrm{sec}$ )

$t_{r a, 0}=$ time when runaway generation via Dreicer process effectively stops (due to $\varepsilon<\varepsilon_{\mathrm{C}}$ )

$t_{t r, 0}=$ time at which trapped percentage reaches $p_{t r a p, o}$

$t_{\text {trap,th }}=$ untrapping time of hot electrons having energy $T_{e o}$. used for normalization of untrapping times

$\mathrm{T}_{\text {eo }}=$ electron temperature in predisruption plasma

$v_{\text {ra }}=$ velocity of runaway

$v_{t} \quad=\left(2 K T_{e} / m_{e}\right)^{1 / 2}=$ thermal electron velocity

$W_{C} \quad=$ critical energy thresholod for runaway generation via Dreicer process

$W_{\text {ra }}=$ energy of runaway

$\delta \mathrm{W}_{\mathrm{r}}=$ energy gain of relativistic electrons required for the generation of one next-generation runaway

$W_{\text {rec }}=$ collisional recoil energy received by plasma electron

$Z_{\text {eff }}=$ effective ion charge

$\alpha\left(Z_{\text {eff }}\right)=W_{C, \text { eff }} / W_{C}=$ correction factor for effective collisional threshold energy $W_{C \text {, eff }}$ vs. $W_{C}$ (presently estimated $\alpha \sim 1-2$ )

$\varepsilon \quad=E / E_{D}=K T_{\theta} / W_{C}=$ electric field normalized to Dreicer field

$\varepsilon_{\mathrm{C}} \quad=$ critical limit of $\varepsilon$ below which direct runaway generation via Dreicer process becomes ineffective (about .025 - .03)

$\varepsilon_{i} \quad=\rho j_{O} / E_{D}=$ initial normalized E-field for $j_{0}$

$\Gamma(\varepsilon) \quad=G_{D} / n_{e}^{f} e e$ 
= normalized runaway generation rate for Dreicer process

(also dependent on $Z_{\text {eff }}$ )

$\rho \quad \cdot \quad=$ Spitzer-Haerm resistivity

$\tau \quad=t^{\prime} / t_{\text {trap, }}$ th

= time after thermal quench normalized to thermal untrapping time

$\tau_{\text {aval }}=2 \alpha m_{e} c \ln \Lambda / e E$

= approximate exponentiation time for collisional avalanching of relativistic runaways

$\tau_{\text {trap }}(W)=$ exponential decay time of trapped high-energy electrons having energy $W$.

\section{INTRODUCTION}

Runaway electrons, often with energies in the many- $\mathrm{MeV}$ range and sometimes carrying a large fraction of the plasma currents, have been observed in essentially all major tokamak devices and also have been analyzed theoretically (Ref.1). Earlier, attention generally has focussed on runaways generated and confined during the normal plasma discharges. Only recently, emphasis has shifted to runaways generated and/or accelerated during disruptive events in such discharges, in particular since it was realized that runaway beams carrying up to about $1 / 2$ the normal current were generated in JET disruptions and may have produced significant damage in that device (Refs. 2); similar observations were made also on TFTR and JT-60 (Refs. 3 and 4). The generation of such runaway beams is not yet fully understood; but, based on these observations, even stronger beams with particle energies ranging up to several hundred $\mathrm{MeV}$ and total beam energies in the 100's MJ range have been projected for ITER, and are presently considered a potentially very serious damage source for that device (Ref. 5) as well as other future large tokamak devices.

In principle, the runaway beams observed during disruptions 
might, of course, simply be remnants of runaway populations already existing before the disruption. However, this appears rather unlikely in general for various reasons: (i) Many otherwise similar nondisruptive discharges generally do not show indications of large runaway beams. (ii) The normal discharge conditions in these high$T_{e}$ devices, in particular their low loop voltage, often are not suited for the generation and acceleration of multi-MeV runaways. (iii) One would expect that most existing runaways generally will be lost from the discharge together with the hot thermal electrons due to the magnetic field perturbations existing during the initial disruption phases. (In TFTR (Ref. 13), this loss process sometimes is seen through the observance of hard-X-ray bursts just at the beginning of a disruption.)

Correspondingly, the present paper will present a systems-type analysis of the potential runaway generation in tokamak disruptions considering various mechanisms of particular importance for disruptions in large devices like TFTR, JET, DIIID and ITER. This investigation will be based on our present understanding of the disruption physics and on the plasma parameters relevant to these large devices; on that basis, it will analyze runaway generation through three different processes, including (i) the "normal" dragevaporation process originally investigated by Dreicer (Ref. 6) and others (Refs. 7 and 8 ), as well as other potential processes that are not normally considered, in particular the generation of runaways (ii) through the acceleration of trapped thermal high-energy electrons that survive the initial open-field-line phase of the disruption, and (iii) through collisions of previously generated runaways with thermal electrons (Refs. 9 and 10). Here, our study will focus mainly on the actual generation of the runaways. Questions of their subsequent acceleration and confinement, including their orbits, beam equilibria etc. - which obviously may have a significant influence on the finally observed runaway beams will be treated only superficially; they will be the target of a subsequent, separate analysis.

The results indicate that processes (i) and (ii) alone may explain the observed strong runaway beams in some cases, with details depending on the actual plasma parameters in the post-thermal- 
quench plasma. In addition, process (iii) can lead to large, avalanche-type enhancements of any existing runaway population, as well as a simultaneous sizable down-shifting of the resulting overall energy spectrum whenever processes (i) and (ii) by themselves will not lead to strong runaway beams. These latter enhancements can be expected to be particularly pronounced for the projected 20-MA discharges in ITER.

\section{BASIC DISRUPTION PICTURE}

As a basis for our subsequent discussions, we will briefly summarize the present, rather widely accepted, overall picture of tokamak disruptions (Refs.11, 12, 13). This picture envisions (see Fig. 1) in "Phase $I^{\prime}$ an initial growth of low $(m, n)$ islands in the hot discharge over an extended period, leading to an overlap and reconnection of these islands. Subsequently, in Phase II, this reconnection then will result in a rapid cooling of the plasma, the so-called "thermal quench", which typically will occur over only a few hundred $\mu \mathrm{sec}$, most likely resulting from a streaming of the hot electrons to the wall along now open field lines. Often, in particular in high-density disruptions, some parts of this thermal quench also may be enhanced and/or even brought about through a radiational instability (Ref.14) triggered by a rapid influx of impurities. Although details are not yet well known, it appears that this process - possibly going through a brief intermediate phase at a few hundred eV (in. the following called "Stage $I^{\prime \prime}$ ) - will bring the plasma temperature down to well below $100 \mathrm{eV}$, probably to around $10 \mathrm{eV}$ ("Stage II").

Also, the current profile generally appears to widen and flatten during the thermal quench (Ref. 13), leading to the well-known small positive (typically up to about $10 \%$ ) spike in the observed plasma current lasting for about 1-2 msec (Ref. 15). In addition, transient current filaments (see Refs. 16 and 17) carrying significantly higher current densities may occur at this point.

During the subsequent "current quench" period, typically lasting from a few to about $20 \mathrm{msec}$, the total plasma current then will decay - mostly resistively with $\mathrm{T}_{e} \sim 5-20 \mathrm{eV}$ (the latter as derived 
by various analyses; no direct measurements seem to exist as yet) either directly to zero, or to a new plateau, "current tail", at a level somewhere between about $10 \%$ and about $70 \%$ of the initial current $\mathrm{I}_{0}$. From that plateau - if so -, the current finally will decay to zero on a time scale of up to several hundred msec. It is generally assumed that the discharge current in the current tail is carried essentially completely by runaway electrons. (Ref. 2)

While recognizing its varinus uncertainties, we think this picture overall can provide a useful reference base for our discussion of potential runaway generation, i.e. of the physics mechanisms bringing these electrons to the critical energies from which they subsequently can actually "run away" to attain the projected multi-MeV energies.

Obviously, a serious question concerns the mechanism that will prevent the newly born runaways from running into the wall along stochastic field lines (as supposedly do the original hot thermal electrons during the thermal quench) before they obtain energies sufficiently large so that their drift orbits are less influenced by magnetic islands (Ref. 18). While a definite answer to this question cannot be given at this point, we notice that runaways actually are observed quite frequently in disruptions, and would like to offer a potential explanation: The original island formation generally is driven - at least in sizable part - by pressure gradients and a current density concentration in the inner, hotter parts of the discharge. After the thermal quench and the respective flattening of the current profile, these reasons for island formation have disappeared, as generally have the low-q resonant surfaces within the discharge. Correspondingly, it could be expected that the existing field perturbations will disappear and reasonable magnetic surfaces be reestablished, probably on a resistive time scale which - near the plasma surface - could be as low as a few hundred $\mu$ sec for $T_{e} \sim 5-10 \mathrm{eV}$. Thus, the new runaways (gaining less than a few $\mathrm{MeV}$ during such time) may be prevented from hitting the wall quite soon after the thermal quench. In addition, it should be noted that at least two of the generation mechanisms discussed below - namely the Dreicer evaporation and the avalanching process - can remain active as long as plasma current densities comparable to the initial ones exist, i.e. generally for a few msec beyond the termination of the thermal quench. In addition, as shown 
below, the de-trapping of trapped high-temperature electrons - in particular in combination with a subsequent avalanching - may be able to generate significant runaway currents for up to a few msec following the thermal quench.

\section{RELEVANT PARAMETER REGIMES FOR LARGE TOKAMAKS}

Table I summarizes the typical operating parameters of various large tokamaks which we will use for obtaining approximate values for various runaway-relevant parameters during disruptions:

The major and minor radii quoted in that Table simply refer to the vacuum vessel; the actual major radii of the plasma generally may deviate by up to about $20 \%$ both ways, while the plasma's minor radii may be smaller by up to about a factor two in some discharges. Similarly, the elongation ratios, b/a, toroidal fields, $B_{t}$, and total currents, Itot, of actual plasma discharges quite often will be less than the maximum values quoted in Table I. The current density during the normal operation phase, of course, will depend on these parameters as well as the peakedness of the discharge: The two displayed current density values - $j_{q}$ for $q(0)=1$, and the "average" current density, $\langle j\rangle=1 / \pi a b$, for a homogeneous current density over the entire vessel cross section - simply are derived from these machine parameters, and generally will bracket the current densities of actual full-current discharges. The presented "typical" values, jo, represent a geometric mean of the other two; quite interestingly, these latter values do not vary widely between the various devices. Finally, actual operational plasma densities, $n_{e}$, in these machines generally will vary over a range of up to a factor 2-5 above and below the "typical" values of Table 1 ; in addition, it should be noted that recent preliminary experimental indications on TFTR as well as DIIID (Ref. 19) point to a general tendency of the observed line-averaged densities to rise during the initial cooling phase of the disruptions - potentially by factors of up to 2-3. 
In the follow." s, these "typical" operating values will be used as reference; any changes in the runaway-relevant parameters due to variations in actual operating parameters, as well as due to the mentioned potential rise in $n_{e}$ during a disruption, can then be derived quite easily from the relevant scaling laws.

The main parameters relevant to the generation of runaways include the electron-electron collision frequency,

$$
f_{e \theta}=n_{e} e^{4} \ln \Lambda /\left[4 \pi \varepsilon_{0}^{2}\left(k T_{e}\right)^{3 / 2} m_{e}{ }^{1 / 2}\right],
$$

of the cold thermal electrons, the electron ion collision frequency, $f_{e o}$ $=Z_{\text {eff }} f_{e e}$, the effective B-parallel electric field component, $E$, and the scale time, $t_{a c c} \sim m_{e} c / e E$, approximating the time needed for a runaway to reach near-relativistic speed. (The actual time needed to reach such speeds generally will be somewhat larger due to a decreasing $E(t)$; however, as will be seen below, $E$ generally is not expected to decrease drastically during the time of initial runaway generation - through processes (i) and (ii), while changes of $E$ during the later avalanche process are less important; thus, the above-defined tacc still provides a good estimate for the relevant time scale.)

Clearly, the various parameters have to be estimated separately for both disruption stages. For this purpose, we assume the mentioned electron temperatures, namely $T_{e}=300 \mathrm{eV}$ and $10 \mathrm{eV}$, and $Z_{\text {eff }}=5$ and 3 for Stages $\mathrm{I}$ and $\mathrm{II}$, respectively, with the latter being roughly consistent with a carbon dominated plasma at the respective electron temperatures. In addition, the quoted values for $f_{e e}$ and $f_{e o}$ assume plasma densities equal to the quoted "typical" operating values.

Concerning the B-parallel electric field component, E, we first note that the quoted electron collision frequencies all are high compared with the rates-of-change of current density etc.. Therefore, the electric field, E, actually seen by the electrons and relevant for runaway generation, is determined best from the electric fields needed for driving the resistive currents, i.e., 


$$
E(x, t)=\rho(t) j_{p}(x, t)
$$

with the current density, $j_{p}(x, t)$, actually carried by the thermal plasma, and the resistivity $\rho(t)$, for which the classical Spitzer resistivity is taken (Ref. 20). As a basic measure of this field, Table I shows the resulting values for $E_{0}=\rho\left(T_{e}\right) j_{0}$ and $t_{a c c}$ for the given "typical" current densities, $j_{0}$, and the assumed values of $T_{e}$ and $Z_{\text {eff }}$. Understandably, the quoted values again vary little between the various devices, but they are very sizably different for the two stages.

\section{ANALYSES OF RUNAWAY GENERATION PROCESSES}

Using the parameter values assumed or derived in Sec. III, we now will estimate the relevant time scales and relative effectiveness for each of the mentioned processes.

\section{Basic Physics Assumptions and Equations}

This analysis will be based on a zero-dimensional analysis of runaway generation in the cold post-thermal-quench plasma under the influence of the $B$-parallel electric field $E=\rho j_{p}$. The resulting runaway electrons are implicitly assumed to be confined within the plasma generating them. This assumption is justified at least during times when reasonably closed magnetic surfaces exist: Since - as outlined below - the time of actual runaway generation via processes (i) and (ii) generally appears limited to sizably less than a msec, the runaways will aquire durine that time not more than about $10 \mathrm{MeV}$, and thus remain quite well bound to the flux surface of their birth. The various questions of actual runaway confinement - including orbital shift, equilibria of runaway beams, etc. - still can be expected to play a serious role during the subsequent current quench phase when the runaways may acquire the mentioned $100-\mathrm{MeV}$-type energies while $\mathrm{I}_{\text {tot }}$ and the confining poloidal field drop in a major way. However, as mentioned before, the present study is to focus mainly on the original 
generation of runaways.

Also, we will neglect microinstabilities potentially generated by the streaming runaways (Ref. 9): While the existence of such instabilities cannot be ruled out a priori in all cases, their occurrence generally does not seem likely in a disruption in view of the low density of the runaways (relative to the plasma density $n_{e}$ ), their rather wide energy spread, and the high collisionality of the cold plasma.

In addition, we will assume that the total current density, jtot, initially fully carried by the cold plasma - will be equal to the quoted "typical" value, $j_{0}$, just before the disruption. In case of current filamentation, any regions with higher $j_{\text {tot }}$ can be expected to constitute particularly strong sources of runaways.

The remaining basic physics ingredients governing the generation of runaways in tokamak disruptions are sketched qualitatively in Fig. 2 and will be specified in more detail below: During the thermal quench, the electric field $E$ first will rise with decreasing $T_{e}(t)$ while $j_{p} \sim j_{t o t}$ $\sim j_{0} \sim$ const. . At some time, $t_{1}$, when $E$ has attained a sufficient value (and the magnetic surfaces have sufficiently closed), effective runaway generation will commence. Due to their increasing population as well as velocity (before reaching relativistic speed) - the new runaways now will take over a continuously rising part, jra, of the total current, $j_{\text {tot }}$. This will reduce the plasma-carried current, $j_{p}=$ $j_{\text {tot }} \mathrm{j}_{\mathrm{r}}$, and the effective $E$-field, $E=\rho j_{p}$; i.e., the generation of new runaways effectively chokes itself. Simultaneously, this same electric field $E$ - being inductively supported by $-\left(\partial \mathrm{B}_{p} / \partial t\right)$ - will determine the decay of the poloidal magnetic field, $B_{p}$, and of the total current density, jot. Obviously, the momentum, $P_{\text {ra }}$, and kinetic energy, $W_{\text {ra }}$, of any runaways at a given time, $t^{\prime}$, will be determined by the electric field, $E(t)$, existing in the time interval between their "birth" as runaways and the time $t^{\prime}$; due to the inductive generation of that field, $P_{\text {ra }}$ and $W_{\text {ra }}$ will depend mainly on the effective change of the magnetic flux, $\delta \Phi$, through the runaway orbit, and thus mainly on the change of the total current, $\delta I_{\text {tot }}$. Introducing the normal inductance of the 
plasma ring and the respective external equilibrium fields, one is led (see Appendix) to an energy gain of approximately 40-70 MeV for each MA of $\delta I_{\text {tot, }}$ with details depending on the plasma's aspect ratio, the minor-radius position of the runaways, changes of the equilibrium field, etc.

Overall, this runaway generation process is governed by the following set of equations:

$$
\begin{aligned}
& E=\rho(t) j_{p} \\
& j_{p}=j_{t o t}-\theta \int_{0}^{00} n_{r a}\left(P_{r a}, t\right) v_{r a} d P_{r a}
\end{aligned}
$$

with $v_{r a}$ denoting the velocity of the runaways, and with their time dependent momentum distribution, $r_{\text {ra }}\left(P_{\text {ra }}, t\right)$, determined by

$$
\left(\partial n_{r a} / \partial t\right)+e E\left(\partial n_{r a} / \partial P_{r a}\right)=G(t) \delta\left(P_{r a}-P_{c}\right)
$$

with the critical momentum threshold, $P_{C}=\left(2 m_{e} W_{C}\right)^{1 / 2}$, defined from the critical energy, $W_{C}$, (Ref. 8 , see also Equ. (3a) below), and with

(iv) $\quad G(t)=\Sigma_{k} G_{k}\left(E, n_{\theta}, T_{e}, t\right)$

denoting the total rate density of generation for new runaways depending on the plasma parameters - made up by the generation rates, $G_{k}$, for the various generation processes (i)-(iii). In addition, jtot $(t)$ and $E$, of course, are coupled through Maxwell's equations to the poloidal magnetic field distribution and its rate of change.

Using (2c) and the equation of motion for the runaways, it is easily shown that the resulting momentum spectrum of the runaways can be expressed by

$$
n_{r}\left(P_{r}, t\right)=G\left(t^{n}\left(P_{r}, t\right)\right)
$$

where $t^{n}$ is the time of "birth" of the runaways that have a momentum 
$P_{r a}$ at the time $t^{\prime}$; in this, $t^{\prime \prime}\left(P_{r a}, t\right)$ has to be obtained from

$$
P_{r a}=P_{c}+\theta_{t^{n}} f^{t^{\prime}} E(t) d t
$$

\section{Analysis of Runaway Processes}

Obviously, the resulting overall runaway generation will depend critically on a quite compisx interplay between the various plasma parameters and their time dependences, in particular on $j_{0}, T_{\theta}(t), n_{\theta}$ etc. as well as the various $G_{k}$ 's. A general analytic solution of these equations determining the important $E(t)$, and the actual runaway distribution clearly is not possible; any fully quantitative analysis will require the numerical treatment of a variety of specific cases as well as a detailed knowledge of the relevant plasma parameters.

In view of this situation, the following analysis of the relative effectiveness and time scales of the various runaway generation processes will be performed for a few idealized cases using the machine and plasma parameters quoted earlier. From this, an overall understanding will emerge from which reasonably correct gross projections of the effective runaway generation may be obtained. Although the three runaway processes (i)-(iii) generally may occur simultaneously, we will analyze them independently, and subsequently combine the results of these analyses to an overall picture.

\section{A) "Normal" Dreicer Evaporation}

As mentioned, the evaporation model of runaway generation, first pioneered by Dreicer (Ref. 6) has since been analyzed by a variety of authors (e.g. Refs. 7 and 8); here, we will essentially follow Ref. 8. All investigations consider the changes in the electronic distribution function occurring in the presence of an electric field $E$, and determine the generation rate of runaway electrons from the net flow of electrons across a critical energy level 


$$
\begin{aligned}
W_{C}= & n_{e} e^{3} \ln \Lambda / 4 \pi \varepsilon_{0}{ }^{2} E \\
& =\left(E_{U} / E\right) k T_{\theta}=k T_{\theta} / \varepsilon
\end{aligned}
$$

containing the "Dreicer field"

$$
E_{D}=n_{\theta} \theta^{3} \ln \Lambda / 4 \pi \varepsilon_{0}{ }^{2} k T_{\theta}
$$

and the normalized electric field

$$
\varepsilon=E / E_{D}
$$

where $m_{e}, e, v_{\text {th }}$ denote the electrons' mass, charge, and thermal velocity $v_{\text {th }}=\left(2 k T_{\theta} / m_{\Theta}\right)^{1 / 2}$, respectively. For electrons above this energy level the electric acceleration will be larger than the average drag from electron-electron collisions. (Actually, this only applies in cases where $W_{C} \ll 511 \mathrm{keV}$ (Ref. 21), which, however, is well satisfied in all cases of interest here except for plasma current densities in the range $j_{p}<a$ few $A \mathrm{~cm}^{2}$ that are quite small compared with those of interest.)

Quite interestingly, using $E=\rho j$ with the usual Spitzer-Haerm resistivity (Ref. 20),

$$
\rho=\left(1 / \gamma_{E}\right) m_{e}{ }^{1 / 2} Z_{e f f} e^{2} \ln \Lambda / 64(2 \pi)^{1 / 2} \varepsilon_{0}{ }^{2}\left(k T_{e}\right)^{3 / 2},
$$

with $\gamma_{E}=.58$ and .78 for $Z_{\text {eff }}=1$ and 4 , respectively, one obtains quite simply

$$
\varepsilon=\left(\pi^{1 / 2 / 16 \gamma_{E}}\right) Z_{e f f}\left(v_{D} / v_{t h}\right) \sim\left(Z_{e f f} / 6\right)\left(v_{D} / v_{t h}\right)
$$

where $v_{D}=j_{p} / e n_{e}$ is the current-induced drift velocity of the thermal electrons.

The resulting runaway generation rates, $\left(d n_{r a} / d t\right)_{D}$, calculated by the various treatments are somewhat different (Ref. 8) - although not really significantly so: within a factor of a few, they are well 
described by the formula

$$
\left(d n_{r} / d t\right)_{D}=G_{D}(\varepsilon(t))=n_{e}^{f_{e e}} \Gamma(\varepsilon)
$$

with the electron-electron collision frequency, $f_{e e}$, and the normalized source strength $\Gamma(\varepsilon)$ which nowadays generally is written in the form (Ref. 8)

$$
\Gamma\left(\varepsilon, Z_{\text {eff }}\right)=K\left(Z_{\text {eff }}\right)(1 / \varepsilon)^{3\left(Z_{\text {eff }}+1\right) / 16} \exp \left\{-(1 / 4 \varepsilon)-\left[\left(Z_{\text {eff }}+1\right) / \varepsilon\right]^{1 / 2}\right\}
$$

where the factor $K\left(Z_{e f f}\right)$ is of order unity. The function $\Gamma\left(\varepsilon, Z_{\text {eff }}\right)$ is reproduced in Fig. 3. Following Ref. $7, K\left(Z_{e f f}\right)=.35$ is chosen here, independent of $Z_{\text {eff }}$. The dashed lines represent the results of the numerical calculations of (Ref.7) as an indication of the differences between the results of the various theories. (As indicated by the difference between the numerical curve for $Z_{\text {eff }}=10$ from that given by (7), a somewhat smaller value of $K$ might apply for $Z_{\text {eff }}=$ 10.) Also, it should be emphasized that $Z_{\text {eff }}$ only enters through Eq.(7), while $\varepsilon$ itself does not contain $Z_{\text {eff }}$.

Of this result, the points most salient for the present analysis are the very rapid rise of $\Gamma(\varepsilon)$ by about 4 orders of magnitude within the range $\varepsilon=.025-.1$ (for $Z_{\text {eff }}=1$ ) and its even faster decrease for $\varepsilon \leq 0.025$ (decreasing by another factor of 30 and $10^{4}$ for $\varepsilon=.02$ and .015 , respectively !). Inserting the actual plasma and time parameters of the experiments under consideration, we find that the runaway current, $j_{r a}$, created through the Dreicer process will not be able to directly contribute a significant part of the overall current, jot, whenever $\varepsilon$ falls below a critical limit $\varepsilon_{C} \sim .025-.03$; i.e., this type of runaway production will effectively cease whenever $\varepsilon<\varepsilon_{\mathrm{c}}$. (Actually, a very similar effective lower limit of $\varepsilon$ also applies to the normal high- $T_{e}$ discharge where the runaway currents resulting from the time-integrated runaway rates still will remain quite small compared with the discharge current - even for discharges lasting several seconds - as long as $\varepsilon<.025$.) Furthermore, we note that, due to the sharp rise of $\Gamma(\varepsilon)$, the curves 
for $Z_{\text {eff }}>1$ - while sizably lower at some points - can be well represented by simply shifting the $\left(Z_{\text {eff }}=1\right)$-curve to larger $\varepsilon$ by multipying all $\varepsilon$ values by about 1.2 and 1.5 for $Z_{e f f}=3$ and 10 , respectively. Therefore, in view of the various theoretical and experimental uncertainties, the various relevant $\varepsilon$-values quoted in our following discussions mostly will refer to the $\left(Z_{\text {eff }}=1\right)$-curve of Fig. 3; for actual cases where $Z_{\text {eff }}>1$, these $\varepsilon$-values will be larger by about 20 to $50 \%$ (for $Z_{\text {eff }}=3$ and 10 , respectively); obviously, this will shift the predicted effects to somewhat higher $\varepsilon$-values, but - due to the steepness of $\Gamma(\varepsilon)$ - otherwise essentially the same basic relationships and results are obtained. (Of course, it is again this very steep rise of $\Gamma$ which renders the mentioned differences between the various theoretical calculations effectively nonsignificant, at least as far as the present analyses are concerned.)

\section{Evaluation for Large Devices:}

To orient the evaluation of this runaway process for large tokamak devices, the size of the relevant parameters for Stages I and II in the various devices are summarized in Table II, including an "initial normalized field",

$$
\varepsilon_{i}=\rho j_{0} / E_{D}
$$

calculated from the typical values of the initial current density, jo, the plasma density, $n_{e}$, and the temperature, $T_{e}$, as quoted in Table 1.

Unfortunately, a general integration of the relevant equations does not seem possible even for this single generation process. Also, - as we will see below - the results will very strongly depend on the relative size of various time scales. Therefore, we will consider and evaluate a number of idealized cases: In particular, these will include - as a starting point - a Group (1) of cases where we assume that the $T_{e}$ values quoted for Stages I and II - together with the respective resistivities $\rho$ - will be attained instantaneously, i.e. on a time scale short compared with the time needed for significant runaway 
generation to occur; subsequently; - in Group (2) of situations - we will consider briefly the consequences of a more realistic slower fall of $T_{\theta}$. In each of the two groups, we will consider three separate values of $\varepsilon_{j}$.

\section{Group (1): Assuming instant drop of plasma temperature:}

Assuming an instant drop in $T_{\theta}$, runaway generation will start with a rate determined by $\varepsilon_{i}$. Analyzing the $\varepsilon_{i}$-values given in Table II, while also noting their variability with the changes of the underlying discharge parameters (in particular densities !) as well as with changes during the thermal quench (apparent density rise!), it appears that quite generally $\varepsilon_{i}<.025$ in Stage I (except possibly for very-lowdensity discharges and/or if $T_{\theta}$ is significantly lower than $300 \mathrm{eV}$ ); thus, the runaway generation via this process during Stage 1 can be expected to be small, i.e., to lead to runaway currents, $j_{r a} \ll j_{0}$.

In Stage II, on the other hand, $\varepsilon_{i}$ may fall into the critical range $\varepsilon_{i}>$ .03 (for $Z_{e f f}=3$ ) for a sizable range of operating parameters - in particular for full-current, low- $n_{e}$ discharges. Although the details again will depend on the mentioned density rise during the thermal quench period, this conclusion appears particularly true for TFTR. The $\varepsilon_{i}$-value for ITER is sizably smaller, making this process less prevalent; however, it appears that densities actually used in ITER discharges may include also values sizably smaller than the rather high "typical" value presently projected for ITER in Table I.

Correspondingly, the following discussions will be limited to Stage II: Assuming that stage is reached suddenly at $t=$ " 0 " with $\varepsilon_{i}>.025$, runaway generation will start from that initial value $\varepsilon_{i}$, and following our earlier argument - the values of $j_{p}, E$ and $\varepsilon$, then will continuously decrease due to the rising $j_{r a}$. Correspondingly, the generation of runaways will continuously decrease, and finally, will effectively cease when $j_{p}$ falls to the critical level, 


$$
j_{p, c r}=\varepsilon_{C}\left(E_{D} / \rho\right)
$$

- at a time "tra,o". Since as mentioned earlier $j_{\text {tot }} \sim j_{0} \sim$ const., we find that the runaways - at that time - will carry a fraction

$$
g_{0}=j_{r a, c r} / j_{O}=\left(\varepsilon_{i}-\varepsilon_{c}\right) / \varepsilon_{i} \sim\left(\varepsilon_{i}-.025\right) / \varepsilon_{i} .
$$

of the original current $j_{0}$. Clearly, this result alone could explain the sizable fractions of runaway currents observed in some disruptions.

For the following discussion of specific cases, we should note first that a very small density fraction of runaways,

$$
P_{r a, j o}=n_{r a, f} / n_{e}=j_{0} / e n_{\theta} c
$$

will be sufficient to carry the full current density jo whenever the runaways attain relativistic speed; taking the quoted plasma densities together with the typical current densities of Table I, we find Pra,jo $10^{-3}-10^{-4}$ (see Table II).

Furthermore, we will have to distinguish the following times (all measured from our starting point ${ }^{n} t=0^{n}$ after the thermal quench):

$t_{r a, c}=$ time at which the runaway population reaches a critical density, $n_{r a, c}=n_{e} g_{0} p_{r a, j o}$, where these runaways, if fully relativistic, would carry the runaway current density $j_{r a, c r}=\left(j_{0}-j_{p, c r}\right)$ that is required to stop further runaway generation; and

$t_{r a, 0}=$ time at which the runaway generation via the Dreicer process actually will stop.

Depending on the relationship of these times to the earlier mentioned acceleration time, tacc, various cases can be distinguished:

(i) Whenever $t_{r a, c}$ is sizably larger than $t_{a c c}$, then most of the runaways will be at relativistic speeds when $n_{r a, c}$ is reached. Thus, further runaway generation actually will stop at that time, i.e. We will 
have $t_{r a, c}=t_{r a, 0}$ with most of the runaways already being relativistic.

(ii) Inversely, whenever $t_{r a, c r} \ll t_{a c c}$, then the velocities of the runaways still will be badly non-relativistic at that point; in extreme cases, the energy of the runaways still might be lingering close to $W_{C}$, so that $v_{r a} \ll c($ say $(1 / 10-1 / 20) c)$. Then, we may have jra $\ll j_{r a, c r}$, and runaway generation still will continue until the runaway current still rising due to the further increases in the population of the runaways as well as their average velocity - will reach the critical value $j_{r a, c r}$. In this case, we may expect $t_{r a, o}$ to be sizably longer than $t_{r a, c r}$.

Clearly, the actual results can be expected to depend strongly on the size of $\varepsilon_{j}$. Therefore, we will focus our following discussions on 3 separate situations distinguished by their values of $\varepsilon_{i}$, namely two extreme cases, (1a) and (1b), with small and high $\varepsilon_{i}$-values, respectively, and one case, (1c), for an intermediate $\varepsilon_{\mathrm{i}}$ :

Case (1a) for low $\Gamma\left(\varepsilon_{i}\right) \sim<(-6)$, i.e. $\varepsilon_{i} \sim<.035$ for $Z_{\text {eff }}=1$ (or correspondingly, $\varepsilon_{i} \sim<04$ for $Z_{\text {eff }}=3$ ): In this case, the time, $t_{r a, j o}$, is relatively long. At the initial level of $\Gamma\left(\varepsilon_{i}\right)$, this time might be approximated by

$$
t_{\text {ra,jo }} \sim g_{o} P_{r a, o} / f_{e e} \Gamma\left(\varepsilon_{i}\right)=N_{o} / f e e
$$

where $N_{0}=g_{0} P_{r a, o} / \Gamma\left(\varepsilon_{i}\right)$ denotes the approximate required number of electron collisions for the runaway population to reach that level.

In view of the rapid decrease of the actual generation rate, this

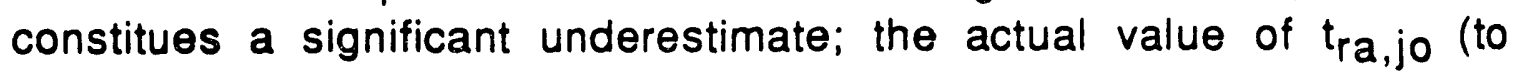
reach, say, $80 \%$ or more of the needed runaway population) can be expected to be a few times longer. For the chosen $\Gamma\left(\varepsilon_{i}\right)<3(-7)$ and $p_{r a, 0}$ $\sim 3(-4), t_{r a, c r}$ can be expected to amount to a few 1000 collision times. Although still amounting only to less than a few $100 \mu \mathrm{sec}$ (at most), the actual times needed to reach $p_{\text {ro }}$ thus often will be easily comparable and even larger than the tacc values of Table $I$. 
Thus, situation quite nearly corresponds to case (i) from above, with further runaway generatioin being stopped at that time. (As a specific example, we might consider the Stage-ll case in TFTR with $\varepsilon_{\mathrm{i}}=.04$ (for $Z_{e f f}=3$ ), i.e., $\Gamma\left(\varepsilon_{i}\right) \sim 3(-7), f_{e e}=1.6(8) \mathrm{sec}^{-1}, n_{e}=4(13) \mathrm{cm}^{-3}, p_{r a, 0}=$ $7(-4)$, and $g_{0} \sim .03$, we get $t_{r a, j o} \sim 10 \mu \mathrm{sec}$. Thus, the actual time for the runaway ponulation to reach the critical level $p_{r a, 0}$ will be roughly 30-40 $\mu \mathrm{sec}$, which is quite comparable tacc $\sim 27 \mu \mathrm{sec}$.)

Due to the shortness of the elapsed time, we actually will still have $j_{\text {tot }} \sim j_{0}$ (as indicated earlier) of which a part, $j_{p} \sim j_{c r} \sim$ (1$\left.g_{0}\right) j_{0}$, still is carried by the cold thermal plasma, while $j_{r a} \sim g_{0} j_{0}$ is carried by relativistic runaways. Subsequently, the remaining $j_{p}$ will decay resistively, while the runaways mainly will gain further energy without increasing their average velocity and current. Thus, assuming for the moment that all runaways remain confined till the end of the resistive phase (corresponding to the start of the "runaway tail" in the observed discharge current), then the current at the beginning of the runaway tail will be roughly equal to the initial runaway current $j_{r a} \sim g_{0} j_{0}$. During that resistive decay phase, the runaways will acquire the very large projected energies of about 30-60 MeV for each MA of decrease between the initial discharge current and the current at the start of the runaway tail. From present, first estimates, a good confinement of these accelerating runaways by the decaying discharge current appears somewhat difficult to understand for current tails of less than about $50 \%$ of $I_{0}$ - which are often observed (except in the presence of fast-changing vertical fields). Clearly, as mentioned before, this final acceleration process will have to be studied in more detail.

Case (1b) for large $\Gamma\left(\varepsilon_{i}\right)>10^{-4}$. i.e. $\varepsilon_{i} \sim>07$ (for $Z_{\text {eff }}=1$ ) : As we can see from Table II, this situation may apply to disruptions of lowdensity discharges in TFTR, possibly also in some of the other machines (depending on the actual rise of $n_{\theta}$ ). Here, $N_{0}=g_{0} p_{r a, f} / \Gamma\left(\varepsilon_{j}\right)$ will be quite small (about $<20$ ). In this case, as seen from Tables I and II, the time $t_{r a, j o}=N_{o} / f e e$ generally will be sizably shorter than $t_{a c c}$, i.e. this situation corresponds to the case (ii) defined earlier. 
From the relevant numbers, $t_{r a, j o}$ will be smaller than $t_{a c c}$ by a factor $x$ which may reach roughly up to $>10-20$. Correspondingly, the generated runaways still are strongly non-relativistic $(v \sim c /(10-20))$, at that point, and further runaway generation will continue until the increasing runaway current reaches the critical level where $j_{p} \sim j_{c r}$. It appears that this limit is attained through the runaway population rising by somewhat less than a factor $\kappa^{1 / 2}$ (due to the falling $\Gamma(\varepsilon)$ ), and its average velocity rising by somewhat more than $\kappa^{1 / 2}$. Correspondingly, the final runaway density percentage, Pra,f, will exceed $P_{r a, 0}$ by a factor $\left(\sim \kappa^{1 / 2}\right)$ with the majority of the runaways still being sizably non-relativistic (roughly by a factor $1 / \mathrm{k}^{1 / 2}$ ). In the extreme, i.e. when $t_{r a, j o} \ll t_{a c c}$, we may get $p_{r a, f} / p_{r a, o}$ quite large, say up to $3-10$, with the runaways still being equally non-relativistic. Subsequently, without further runaway generation, the remaining resistive plasma current will be further converted quickly into beam current through acceleration of the runaways, until the full discharge current is carried by the runaway beam. In view of the fact that this will necessitate only a velocity increase by a factor $\varepsilon_{j} /\left(\varepsilon_{i}-.025\right)=$ $j_{o} /\left(j_{0}-j_{C}\right)$ (generally < 1.5 or so), we may expect that the runaways still may be sizably non-relativistic even at that point, i.e., when the entire discharge current is tranferred to the runaway beam. Pending our further studies, we may expect that the subsequent development of the generated runaway beam will depend on the gross dynamics of the decaying discharge, with various diverging sequences appearing possible. However, in view of the actually quite fall of $T_{e}$, this case probably may be realized only rarely in practice and thus not be important in reality (see later discussions).

$$
\text { Case (1c) for intermediate } \Gamma\left(\varepsilon_{j}\right) \sim 10^{-5} \text { i.e. } \varepsilon_{j} \sim 05 \text { (for } Z_{\text {eff }}=1 \text { ): }
$$

This case initially will develop similar to case (b): a large fraction of the runaways will still be non-relativistic, but near-relativistic, when runaway generation stops (i.e. when $\varepsilon \sim \varepsilon_{\mathrm{C}}$ ); however, a large fraction of the runaways subsequently may reach relativistic speed before all of the remaining plasma current is transferred to the runaways. The final runaway beam will carry a current somewhere beween $g_{0} l_{0}$ and the full $\mathrm{I}_{\mathrm{O}}$. The runaways then may be accelerated into the many-MeV range 
during the resistive quench of the still remaining (if so) plasma current. Thus, the finally emerging runaway tail could be expected to show currents up to nearly the full current $I_{0}$ carried by many-MeV electrons. However, the final decay of this tail including the potential $100-\mathrm{MeV}$-type acceleration of electrons again will have to be studied further.

It should be noted that in cases (1b) and (1c) the time $t_{r a, j o}$ during which runaway generation occurs is shorter than in case (1a), so that the approximation $j_{\text {tot }} \sim j_{0}$ again is vary valid throughout.

Group (2): Finite cooling time of $T_{\odot}$ :

Finally, we will consider changes in the above conclusions related to the finite actual cooling times of the plasma when approaching Stage II. Obviously, these changes will sizably depend on the comparative time scales of: (i) the assumed, although not yet well defined, thermalquench cooling time, $t_{q u} \sim$ "a few hundred $\mu \mathrm{sec}^{\text {" }}$ - which determines the rise in resistivity and thus the build-up of the resistive field $E$ - , and (ii) the time needed for the evaporative development of a sizable runaway beam which will take over some of the plasma current and thus diminish the resistive E-field. (iii), we should note that $t_{q u}$ generally is larger than tacc.

Since the time scale (ii) will depend drastically on $\varepsilon$, we will again discuss separately the three cases distinguished earlier. The earlier used parameter, $\varepsilon_{i}=\rho\left(T_{e} \sim 10 \mathrm{eV}\right) \mathrm{j}_{\mathrm{o}} / E_{D}$, now may no longer be reached actually in the process, but it still retains significance as the maximum possible value of $\varepsilon$; clearly, and in agreement with our earlier conclusion, there still will not be any significant runaway generation if $\varepsilon_{i}<.025$. Cases (2a),(2b) and (2c) are to refer to the same values of $\varepsilon_{i}$ as the above cases (1a), (1b) and (1c), respectively.

Case (2a): According to our earlier analysis, the time scale for the development of the runaway beam in this case certainly is longer than $t_{a c c}$, and - generally - quite comparable to or even longer than tqu. 
Thus, our earlier fast-drop assumption for $T_{e}$ appears reasonably exact in general, and the earlier results should still hold approximately; in particular:

(i) essentially all of the runaways will be at relativistic speeds when the evaporative runaviay process effectively stops, and

(ii) the total strength of the final evaporative runaway beam current, $\mathrm{j}_{r}$, after the resistive decay of the remaining plasma current will be roughly as given in Equ. (8), i.e., $j_{r a} \sim g_{0} j_{0}$, and this runaway beam may attain the envisioned $100-\mathrm{MeV}$-type energies in the subsequent resistive quench;

(iii) the entire evaporative runaway gene ation will occur within a few $100 \mu \mathrm{sec}$ to at most a msec (so that still $j_{\text {tot }} \sim j_{0} \sim$ const. during the generation of the runaways).

Case (2b): In this case, the earlier derived times for the runaway population to reach $p_{r a, o}$ as weil as those needed for cutting off further runaway generation generally are sizably shorter than tqu . Thus, our earlier assumption clearly will break down - except possibly if the risetimes of $E$ actually are much faster, e.g. via a fast current filamentation process. Instead of $E$ and $\varepsilon$ advancing "suddenly" to their full potential values, $E_{i}=\rho j_{0}$ and $\varepsilon_{i}$, the actual dependencies $E(t)=$ $\rho(t) j_{p}(\hat{\imath})$ and $\varepsilon(t)$ now will be determined by an interplay between the rising $\rho(t)$ and the emerging runaway currents. In particular one can expect the following sequence:

(i) $E(t)$ and $\varepsilon(t)$ first will increase according to the $T_{e}(t)$ of the thermal quench until $\varepsilon \sim .025$;

(ii) subsequently, the further increases of $E(t)$ and $\varepsilon(t)$ driven by the continuing drop in $T_{\theta}(t)$ will be strongly mitigated by the reduction of the plasma current $j_{p}(t)=j_{0}-j_{r a}(t) d u e$ to the developing runaway current jra $(t)$;

(iii) then, $E$ and $\varepsilon$ will reach maxima - where the further rise of these parameters driven by the falling $T_{e}$ is balanced by the increasing $j_{r a}(t)$; clearly, this generally will occur somewhere at intermediate values of $\varepsilon$ (see Case (1c)) where the time scales for runaway production and acceleration become comparable to $t_{q u}$; 
(iv) finally, $E$ and $\varepsilon$ will fall again, and evaporative runaway generation will stop when $\varepsilon \sim .025$.

The details of this process and the resulting final runaway distribution will depend on the details of $T_{e}(t)$ etc. and will have to be analyzed numerically. However, since generally $t_{a c c}<t_{q u} \sim$ few 100 $\mu \mathrm{sec}$, it appears that a major part of the runaways again will have reached relativistic speeds when $\varepsilon$ again has decayed to $\varepsilon \sim \varepsilon_{C}$. Thus, depending on the ratio $t_{a c c} / t_{q u}$, the resulting runaway beams probably are comparable to the results projected earlier for the Cases (1a) or (1c).

Intermediate Case (2c): With the $\varepsilon_{i}$ values reduced from case (2b), but still with $t_{a c c}<t_{q u}$, the final runaway beam will be even closer to that of Case (1a).

In all these cases, the results will be shifted more towards those of Cases (1c) or (1b) with instaneous $T_{e}$ drop, if the effective timescale of the final thermal quench to $10 \mathrm{eV}$ turns out to be significantly faster than the presently projected few $100 \mu \mathrm{sec}$, or in case of fast filamentations.

\section{Summary for Dreicer Process:}

Overall, we conclude that the Dreicer process by itself can lead to relativistic runaway beams carrying a significant fraction of the initial discharge current, $I_{0}$, whenever $\varepsilon_{i} \sim>.025$; and, that this situation may occur for a significant range of full-current-low-tomedium- $n_{\theta}$ discharges in all present large tokamaks - even if the mentioned density build-up in disruptions is as projected. However, this $\varepsilon$-limit generally will not be reached in most high- $n_{e}$ discharges. In ITER, this process appears less effective in view of the projected lower $j_{0}$ values and the generally higher $n_{e}$ in that device; however, the required $\varepsilon$-limit still may be reached even in that device in discharges with lower-than-projected $n_{e}$. 
Furthermore, as indicated, whenever evaporative runaway generation occurs, most of this generation is expected to occur during the first few hundred $\mu \mathrm{sec}$ following the thermal quench and the reclosure of the magnetic surfaces. During that time, the energy gained by the runaways generally will be in the low-10-MeV range; the main energy gain normally will occur during the subsequent current quench which is to be investigated separately later.

(B) Bunaway Generation from the De-Trapping of Trapped HighIemperature Electrons Surviving the Thermal Quench

According to our earlier disruption model, the thermal quench - at least in major part - results from the escape of the hot thermal electrons to the wall by streaming along the now open field lines. Although possibly. needing some smaller modifications (e.g. including a bubble-like gross motion (Ref. 13) of the inner plasma parts to the surface which would reduce the required radial magnetic fluctuation fields, $\delta B_{r}$, to a level nearer to the observed levels), this picture basically appears well founded. However, it obviously applies only to passing hot electrons, while trapped hot electrons may be retained in the disruptions. If these hot trapped electrons are not lost in some other way (e.g. by diffusion which does not seem liklely) some of them may survive in the trapped state until after the re-establishment of magnetic surfaces. In that case, these electrons generally will run away whenever they become untrapped since their energy, $W_{e}>T_{e o}$, easily exceeds the rather low critical energy, $W_{c}<1 \mathrm{keV}$ at $T_{e} \sim 10 \mathrm{eV}$. (With $T_{e o}$ denoting the hot temperature of the pre-disruption plasma, the condition, $W_{C}<$ few $T_{e 0}$, sometimes may be satisfied also under the conditions of Stage $I$; i.e., runaway generation via untrapping could occur already in Stage $I$, if against general expectations - Eurface reclosure becomes effective that early.)

In order for this process to be directly important (i.e. without the additional avalanching considered below), the percentage of trapped electrons, $p_{\text {trap }}\left(t_{c}\right)$, still remaining at the time, $t_{f}$, of field closure has 
to be at least comparable to the earlier defined ratio $p_{r a, 0}$, i.e. We need

$$
p_{\text {trap }}\left(t_{f}\right)=n_{\text {trap }} / n_{e} \sim P_{\text {ra }, 0}
$$

For a first estimate of the potential effectiveness of that process, we will now consider the purely collisional decay of the trapped hot electron population: According to Ref. 22, the lowest-order eigenmode of an electron population of energy $W$ trapped by a mirror ratio, $R_{m}$. will decay exponentially due to collisions with the ions and electrons of the cold post-thermal-quench background plasina. (This projection neglects the energy diffusion of the electrons, whici however will not significantly change the results for most situations.) While the various calculations vary by about a factor 2 (Ref.22), the exponential decay constant, $\tau_{\text {trap }}(W)$, for an electron having an energy, $W$, trapped in a field with mirror ratio, $R_{m}$, is approximately equal

$$
\begin{aligned}
\tau_{\text {trap }}(W) \sim & 3.5 \log _{10}\left(R_{m}\right) 4 \pi \varepsilon_{o} 2 W^{3 / 2} m_{e}{ }^{1 / 2 /\left(Z_{e f f}+1\right) n_{e} e^{4} \ln \Lambda} \\
& \sim 1.5 \ln \left(R_{m}\right)\left(W / k T_{e o}\right)^{3 / 2 /\left(Z_{e f f}+1\right) f_{e e}\left(T_{e o}\right)}
\end{aligned}
$$

with the electron-electron collision frequency, $f_{e e}\left(T_{e o}\right)$, at the temperature, $T_{e 0}$, although with the $\ln \Lambda$ and the $Z_{e f f}$ of the cold plasma. Taking the effective mirror ratio of a flux line having a minor radius $r$

$$
R_{m} \sim 1+2(r / R)
$$

one obtains approximately

$$
\begin{aligned}
& \tau_{\text {trap }}(W) \sim 3(r / R)\left(W / k T_{e 0}\right)^{3 / 2} /\left(Z_{e f f}+1\right) f_{e \theta}\left(T_{e o}\right) \\
& =\left(W / k T_{e o}\right)^{3 / 2} \tau_{\text {trap,th }}
\end{aligned}
$$

with

and

$$
t_{\text {trap, }}=3(r / R) t_{2}\left(T_{e 0}\right)
$$

$$
t_{2}=1 /\left(Z_{e f f}+1\right) f_{e e}\left(T_{e o}\right) .
$$


The total initial population of the lowest-order mode at an energy, $W$, will be approximately equal to the population fraction of thermal electrons having an energy $W$ that is trapped in the pre-thermal-quench discharge. The fraction, Ptrap, 0 , of trapped electrons will be independent of $W$, but will vary with the minor radius due to its dependence on the mirror ratio, $R_{m}$. In the following, we will assume a "typical" value

$$
P_{\text {trap,o }} \sim 30 \%
$$

Assuming a fully thermalized hot-electron distribution in the initial pre-disruption plasma, we then find that - at a time $t^{\prime}$ after the untrapped electrons have left - the total percentage, $p_{t r a p}\left(t^{\prime}\right)$, of still remaining trapped electrons (relative to the initial density $n_{e}$ ) will be approximately giver by

$$
\begin{aligned}
p_{\text {trap }}\left(t^{\prime}\right) & =n_{\text {trap }}\left(t^{\prime}\right) / n_{e}= \\
& =p_{\text {trap }, 0}(4 / \pi)^{1 / 2} \int_{0}^{00} x^{1 / 2} \exp \left[-x-\left(\tau / x^{3 / 2}\right)\right] d x \\
& =p_{\text {trap }, 0} F(\tau)
\end{aligned}
$$

with.

$$
F(\tau)=(4 / \pi)^{1 / 2} \int_{0}^{00} x^{1 / 2} \exp \left[-x-\left(\tau / x^{3 / 2}\right)\right] d x
$$

where $x=W / k T$, and where the time $\tau=t^{\prime} / t$ trap, th is normalized to the untrapping time, $\tau_{\text {trap,th }}$, for a thermal electron at $W=T_{e o}$.

For evaluation, we calculated the function $F(\tau)$ numerically for values $\tau \leq 100$; for $\tau>50$, an asymptotic approximation (good to $<20$ $30 \%$ ) was obtained through first expanding the integrand of Eq.(19) around the minimum, $x_{m}=(3 \tau / 2)^{2 / 5}$, of the exponent $\left(x+\tau / x^{3 / 2}\right)$, and then integrating it, with the result:

$$
F(\tau>100) \sim 2(8 / 15)^{1 / 2}\left(x_{m}^{3 / 2 / k^{1 / 2}}\right) \exp \left[-\left(x_{m}+\kappa\right)\right]
$$

with 


$$
\kappa=\tau / x_{m} 3 / 2
$$

The numerical results together with those of Eq. (20) are graphed in Fig. 3.

The critical lower limit of trapped-electron percentage given by Eq. (13), is reached at a normalized time, $\tau_{f, 0}$, when

$$
F\left(\tau_{f, 0}\right) \sim p_{\text {ra }, o} / p_{\text {trap }, o}
$$

Whenever the actual field closure time, $t_{f}$, exceeds the limit, $t_{f, 0} \sim \tau_{f, 0} t_{t r a p, t h}$, the number of trapped electrons quite rapidly will become insufficient to directly create strong runaway beams.

The normalized $\tau_{f, 0}$-values relevant to the various machines are given in Table III together with the corresponding absolute critical time $t_{f, 0}=\tau_{f, 0} t_{t r a p, t h}$. As mentioned, a typical value of $p_{t r a p, 0}=30 \%$ was used for deriving the quoted $\tau_{f, 0}$-values; due to the steep decay of $F(\tau)$, the influence of realistic variations in $p_{\text {trap,o }}$ over the plasma cross section is not really significant at this point (except very near to the magnetic axis). On the other hand, the change of the scale time, $t_{\text {trap,th }}$ over the plasma cross section - due to its proportionality with ( $r / R)$ - will be more serious (being longest for $r=a$ ); the values given in Table III assume a typical radius, $r=(2 / 3)$ a.

Overall, considering the results of Table III, it appears that this process normally will be directly effective - i.e. without additional avalanching - in disruptions of discharges with a predisruption $T_{\theta}<5$ $\mathrm{keV}$ only for rather short magnetic reclosure times, $\mathrm{t}_{f}<.5 \mathrm{msec}$ after the thermal quench, except possibly for very low $n_{e}$. (This result is particularly true if we accept a further density rise of a factor 2-3 during the disruption.) However, the permissible reconnection times will reach into the more realistic msec range in disruptions of discharges with $T_{\theta} \sim 15 \mathrm{keV}$, even at typical $n_{\theta}$ values. Correspondingly, this process generally may not be really significant by itself in the present devices; however, it may become so in high- $T_{e}$-medium- $n_{e}$ discharges in ITER - depending on the actual reclosure times. Obviously, 
the effectiveness of this process also will be increased whenever the high-energy tail of the electron distribution (in particular around or above $\left.x_{m}{ }^{\top} e_{e 0} \sim(8-10) T_{e 0}\right)$ is enhanced, e.g. through current drive, if at least a part of these electrons becomes trapped. In addition, the runaway generation via this process generally will be stronger at the outer radii, $r$, of the disruption rather than the center; as mentioned, our estimates assumed $r / a=2 / 3$. Correspondingly, this runaway generation also will depend on the radial temperature profile, $T_{e o}(r)$, and may be influenced by any bubble-like motion.

Finally, we might note already, that the collisional avalanching discussed in the next section may sizably reduce the sensitivity limit Eq. (13), and thus extend the permissible delay time, $\tau_{0}$, between the thermal quench and the magnetic reclosure.

\section{(C) Collisional Avalanching of Runaways}

As indicated earlier, any thermal plasma electron aquiring a recoil energy $W_{\text {rec }}>W_{C}$ from a close collision with an existing runaway will have a good probability of becoming a new secondary runaway. Since this generation is proportional to the number of already existing runaways, it can lead to an exponential build-up of the runaway population. A more cursory earlier study (Ref. 9) - based on an investigation of the Focker-Planck equation - judged this process "negligible". Actually, however, this process can play a very important role in large-tokamak disruptions (Ref. 10). Re-analyzing and evaluating the process in more detail through considering directly the total cross section for the creation of a recoil electron with an energy $W_{\text {rec }} \geq W_{c}$ in close collisions between existing runaways and cold plasma electrons, and assuming $W_{c} \ll m_{e} c^{2}$, we found a collisional generation rate for runaways

$$
\left(d n_{r a} / d t\right)_{\text {coll }}=\left(e E / 2 m_{e} \ln \Lambda\right)\left[1 / \alpha\left(z_{e f f}\right)\right]\left(1 / v_{r a}\right) n_{r a}
$$

with $v_{r a}, n_{r a}$ denoting, respectively, the velocity and density of the 
existing runaways; the factor $\alpha\left(Z_{e f f}\right)$ (determining the effective cutoff energy, $\alpha W_{C}$, for collisional runaway generation) presently is estimated between $1-2$ for $Z_{\text {eff }}=1-5$, but this will need to be determined more carefully. As shown in Ref. 10, the number of next-generation runaways produced by each runaway before it reaches relativistic velocities actually is quite small (about $\leq .1$ ). However, after becoming relativistic, each runaway will collisionally generate one new nextgeneration runaway for each energy

$$
\delta W_{r}=2 m c^{2} \alpha\left(Z_{e f f}\right) \ln \Lambda \sim 15 \alpha[M e V]
$$

it gains from the electric field (independent of any simultaneous energy losses the runaway may encounter during this time, e.g. through synchrotron radiation!). As indicated by $\mathrm{Eq} .(23), \delta W_{\mathrm{r}}$ is largely independent of $E$ and of all plasma parameters (except through the dependencies of $\ln \Lambda$ and $\alpha$ ); this independence will break down, and $\left(d n_{r a} / d t\right)_{c o l l}$ will disappear only, whenever $W_{c} \sim m_{e} c^{2}$. That latter condition - simultaneously leading also to the collisional slowdown of any already existing relativistic runaways - can be satisfied quite easily in some high- $T_{\theta}$-high- $n_{\theta}$ regimes of normal large-tokamak discharges; however, in a post-thermal-quench disruption it will occur only for uninterestingly small plasma current densities $j_{p}<$ few $\mathrm{A} / \mathrm{cm}^{2}$.

Whenever the runaways are fully confined, such collisional multiplication will lead to an exponential rise of the runaway population in a plasma

$$
n_{r a}(t) \sim n_{r a, 0} \exp \left(t / \tau_{\text {aval }}\right)
$$

with the exponentiation time, $\tau_{\text {aval }} \sim 2 \alpha \mathrm{m}_{\theta} \mathrm{c} \ln \Lambda / \theta E$, being equal to the time during which a relativistic runaway picks up the energy $\delta W_{r}$. Of course, an incomplete confinement of the runaways will lead to a reduction of this growth rate. Assuming an average confinement time $\tau_{\text {conf, one obtains an effective growth time, }}$ 


$$
\tau_{\text {eff }} \sim \tau_{\text {aval }} \tau_{\text {conf }} /\left(\tau_{\text {conf }}-\tau_{\text {aval }}\right)
$$

which, of course, becomes negative whenever $\tau_{\text {conf }}<\tau_{\text {aval }}$.

Obviously, the runaways generated by this process will contribute as any others to the total runaway current reducing the remaining plasma current, $j_{p}$. However, this multiplication process will stop only when $W_{c} \sim m_{e} c^{2}$, i.e. when the thermal plasma current, $j_{p}$, is reduced to a few $A / \mathrm{cm}^{2}$.

Overall, the following conclusions can be drawn regarding the effectiveness of the collisional avalanching in post-thermal-quench disruptions:

(a) Iime of avalanching: Generally, $\tau_{\text {aval }}$ will be longer than the initial few hundred $\mu \mathrm{sec}$ after the thermal quench where processes (i) and (ii). are most active; i.e., the energy gain of runaways during that time is smaller than or at most comparable to $\delta W_{r}$. Correspondingly, relatively little avalanching will occur during that time; most of the avalanching will occur during the subsequent current quench phase.

(b) Size of avalanche enhancements: Avalanche enhancements of the runaway population during the current quench phase of the disruption can be very sizable. According to the general estimate of (30-50) $M E V / M A$, the runaways may gain during that phase as much as (2-3) $\delta W_{r}$ for each MA of decrease in ltot. Thus, if properly confined, the population of relativistic runaways will grow by up to about an order of magnitude for every $(1-1.5) \mathrm{MA}$ of $\delta 1_{\text {tot. }}$. While the confinement question will have to be studied in more detail later, it appears at present that the orbits of accelerated runaways normally might be confined in the decaying disruption quite well until $I_{\text {tot }}$ decays to about (1/2) - (1/3) of the value it had when the respective runaways were "born". Thus, in the existing large tokamak devices having $I_{\text {tot }} \sim$ few MA, the avalanche growth rates generally will not be significantly reduced by nonconfinement of the runaways. Correspondingly, collisional avalanching may increase the runaway population originally generated by the other 
processes by a factor of $100-1000$ in the existing devices.

In a device like ITER with $I_{\text {tot }} \sim 22 \mathrm{MA}$, our analysis leads to even larger avalanche factors of up to roughly $10^{10}-10^{15}$ - if the avalanche process cannot be properly suppressed. Since about $2 \times 10^{10}$ $\mathrm{cm}^{-3}$ of relativistic runaways will suffice to carry the usual 100 $\mathrm{A} / \mathrm{cm}^{2}$, an exceedingly small initial runaway population (a few per $\mathrm{cm}^{3}$, or even less!) remaining from the original discharge, or created by other the processes may thus be amplified into runaway beams carrying sizable fractions of the initial plasma current.

Since avalanching simply will amplify the runaway populations generated by the other processes (as well as any runaways surviving the initial magnetic disturbances), it will effectively widen the ranges of plasma parameters for which strong runaway beams may be expected due to the other processes-plus-avalanching. In presently existing machines (TFTR, JET, DIID etc.), the critical minimum field, $\varepsilon_{C}$, for which runaway generation through the evaporative process can be observed, generally will be shifted down to about $\varepsilon_{\mathrm{C}} \sim .02$ (for $Z_{\text {eff }}=$ 1 ), in more extreme cases maybe down to about .015. This will widen the range of operating parameters where disruption runaways can be expected; however, considering the range of $n_{e}$ and $T_{e}$ generally encountered in these devices, such a change still may not be really important overall. In ITER, however, avalanching may shift $\varepsilon_{C}$ down to $.006-.01$, so that sizable runaway beams may result even close to or at its projected large "typical" densities, $n_{\theta}$. Thus, it can be expected that runaway beams may prove to be much more common in ITER than in the smaller devices.

For runaway seeding via trapped thermal electrons - if such trapped electrons can survive the thermal quench at all -., the estimated avalanche enhancements will lengthen the permissible time limit for reconnection by a factor of $1-2$ over the values given in Table III for the present machines. In even larger machines, like ITER, avalanching could easily stretch the time limit to several msec, so that runaway generation through untrapping and subsequent avalanching could become quite universal, except if the times needed for cooling and re- 
connection will be much larger than that.

(c) Change in energy spectrum: Whenever such avalanching occurs, the energy spectrum of the runaways will be strongly influenced. Assuming full confinement and no energy losses of the runaways, the energy distribution of the runaways will be about proportional to $\exp \left[-W / \delta W_{c}\right]$ up to a maximum energy, $W_{\text {max }}$, which is determined by $\delta l_{\text {tot }}$; in a more realistic runaway confinement, the distribution may be cut off at a lower maximum energy and/or the exponential decrease may be sharpened (depending on the confinement details). In all cases, however, the majority of the runaways will be at quite low energies $\leq 10-20 \mathrm{MeV}$.

(d) Changes in spatial distribution: Avalanching also can seriously change the spatial distribution of the runaways in the decaying disruption: Since the collisional generation of runaways will preferentially occur in the higher-density center of the discharge, and since the drift-related outward shift of $15-25 \mathrm{MeV}$ electrons normally is quite small in the large tokamaks of interest here, it appears that the overall outward shift of the resulting total runaway beams could be much smaller than the increasing energy $W_{r}$ of the initial seed runaways would require. Obviously, this result in particular together with (c) - might explain the quite frequent observation of apparent runaway damage on the inside limiter which is quite difficult to understand for 100's-MeV-type runaways.

(e) Influence on runaway current tails: It appears that avalanching also could seriously influence the decay and behavior of the runaway tails of the disruption whenever the decay rates become sufficiently large so that $\delta W_{c}<m_{\theta} c^{2}$. Clearly, this effect will require further attention in our subsequent study.

\section{SUMMARY}

Overall, the results may be summarized as follows:

(i) The usual evaporative "Dreicer" process by itself can quite easily generate sizable runaway beams in the existing devices, in 
particular during disruptions of high-current-medium-to-low- $n_{\Theta}$ discharges. In ITER, such disruption runaways are expected to be limited mostly to discharges with $n_{\theta}$ values sizably smaller than the projected typical ones.

(ii) The effectiveness of runaway generation through the untrapoing of trapoed hot-thermal electrons remaining from the pre-thermal-quench plasma will depend on the actual timed delay between the thermal quench and the reclosure of the magnetic field lines. In general, we expect that this process will not be important by itself in the present devices having $\mathrm{T}_{e 0}<5 \mathrm{keV}$; however, it might become effective in disruptions of the high- $T_{e}$ and/or low- $n_{e}$ discharges expected for ITER.

(iii) Both processes, (i) and (ii), will occur and be completed mostly during the initial few $100 \mu \mathrm{sec}$ after the thermal quench.

(iv) During the subsequent resistive quench period, collisional avalanching generally will generate a pronounced exponential growth of any runaway populations generated by processes (i) and/or (ii) and/or others, with an order-of-magnitude growth in runaway population generally occurring for about each 1-1.5 MA of drop in the discharge current. If sufficient numbers of runaways are generated initially, then this process will proceed until the resulting runaway beam will carry essentially all of the remaining discharge current. Maximum expected avalanche factors of up to $10^{2}-10^{5}$ in the present devices will only slightly extend the range of operating parameters for which runaways may be expected; however, the very large avalanche factors of up to $10^{10}-10^{15}$ - if avalanching is not properly suppressed - expected for ITER may lead to strong runaway beams over a sizable range of disruption parameters, except possibly those at particularly high densities.

(v) While strongly enhancing the strength of any runaway beams, avalanching will shift the main part of their energy spectrum down to relatively low energies around $10-20 \mathrm{MeV}$. Also, it may significantly influence the position of the runaway beams within the disrupting discharge. 
The behavior and decay of the generated runaway beams is to be investigated in a separate study.

\section{ACKNOMLEDEMENTS:}

A large part of this work was performed at PPPL during two summer and part-sabbatical visits of one of us (H. H. F.) with the financial support by PPPL. The authors would like to express their thanks in particular to Drs. K. M. Young, D. E. Post, K. M. McGuire, and D. M. Meade for their kind support and encouragement. Also, we would like to thank them as well as many other PPPL personnel, in particular Drs. M. G. Bell, E. D. Fredrickson, K. W. Hill, A. C. Janos, D. W. Johnson, D. Mansfield, S. Medley, R. Nafzikian, and J. F. Schivell, and Dr. A. G. Kellman of GA Technologies for numerous conversations contributing to the paper. The work was performed under the auspices of the Department of Energy under Contracts DE-AC02-76-CHO-3073 and, in part, DE-FG02-92ER54183. 


\section{BEFERENCES}

(1) For a review of early data, see H. Knoepfel and D. A. Spong, Nucl. Fusion, 19, 785 (1979);

(2) O. N. Jarvis, G. Sadler, and J. L. Thompson, Nucl. Fusion 28, 1981 (1988)

(3) G. W. Barnes, A. Janos, D. Losser, D. K. Owens and M. Ulrickson, Fusion Technol., 19, 1761 (1991)

(4) H. Takatsu et al., Proc. Symposium on Nuclear Fusion Technology, Tokyo (1988);

(5) K. A. Niemer, C. D. Groessmann, J. G. Gilligan, and H. H. Bolt, Proc. 13th Symposium on Fusion Engineeering, Knoxville, TN, October 1989, p. 941; and A. J. Russo, Nucl. Fusion 31, 117 (1991);

(6) H. Dreicer, Phys. Rev. 117, 329 (1960);

(7) R. M. Kulsrud, Y. C. Sun, N. K. Windsor, and H. A. Fallon, Phys. Rev. Lett. 31, 690 (1973);

(8) V. V. Parail and O. P. Pogutse, in REVIEWS OF PLASMA PHYSICS, Vol. 11, Consultants Bureau, New York, 1986, p. 1;

(9) E. R. Harrison, Nucl. Energy, Part C, 1, 105 (1960) and ibid. 4, 7 (1962);

(10) R. Jayakumar, H. H. Fleischmann, and S. J. Zweben, to be published in Phys. Letters;

(11) K. McGuire et al., J, Nucl. Mater. 121, 329 (1984);

(12) J. A. Wesson, R. D. Gill, et al., Nucl. Fusion, 29, 641 (1989);

(13) E. D. Fredrickson, K. McGuire, M. Bell, C. E. Bush, A.Cavallo, R. 
Budny A. Janos, D. Mansfield, Y. Nagayama, H. Park, J. Schivell, G. Tayior: M. C. Zarnsdorff, and J. Drake and R. Kleva, Princeton Plasma Physics Laboratory Report, PPPL 2785 (January 1992); and E. D. Fredrickson, K. McGuire et al., Rev. Sci. Instr. 59, 1797 (1988);

(14) P. E. Stott, Nucl. Fusion, 28, 1469 (1988); and A. Gibson, Nucl. Fusion, 16, 546 (1976);

(i5) J. A. Wesson, D. J. Ward, M. N. Rosenbluth, Nucl. Fusion 30, 1011 (1990);

(16) A. Bondeson, P. D. Parker, M. Hugon, and P. Smeulders, Nucl. Fusion, 31, 1695(1991);

(17) G. Waidmann and G. Kuang, Nucl. Fusion 32, 645 (1992);

(18) S. J. Zweben. B. V. Waddell, D. W. Swain, and H. H. Fleischmann, Nucl. Fusion, 20, 477 (1980); and H. E. Mynick and R. E. Duvall, Phys. Fluids, B1, 750 (1980);

(19) A. G. Kellmann, private communication.

(20) L. Spitzer, Jr., Physics of lonized Gases, Interscience Publishers, New York, 1962 (p. 139);

(21) J. W. Connor, R. I. Hastie, Nucl. Fusion, 15, 415 (1975);

(22) R. F. Post, Nucl. Fusion, 27, 1577 (1987). 


\section{Eigure Captions:}

FIG.1: Qualitative changes of discharge current, $I(t)$, and electron temperature $T_{\theta}(t)$, during a disruption. (Time window shown is of the order of 10-20 msec)

Fig. 2 Qualitative time dependencies of total current density, $j_{t}$, plasma current, $j_{p}$, the B-parallel electric field, $E$, and the current densities, $j_{p}$ and $j_{r a}$, carried by the cold plasma and the generated runaways, respectively.

Fig. 3 Normalized runaway generation rate, $\Gamma(\varepsilon)$, via Dreicer evaporation from Eq. (7) (Ref. 8) for $Z=1,3,10$. Dashed lines denote numerical results from Ref. 7 .

Fig. 4 Normalized decay function $F(\tau)$ for percentage of trapped post-quench thermal electrons.

\section{IABLECAPTIONS:}

TABLE I: Basic Plasma Parameters for Large Tokamak Devices

TABLE II: Normalized parameters and relativistic-runaway percentage, Pra,o, required for carrying full jo (see Eq. (11)) - for various machines as derived from parameters of Table $\mathrm{I}$.

TABLE III: Parameters relevant for runaway generation via trapped hot thermal electrons. 
IABLE]:

PARAMETERS

TFTR JET DIIID ITER

Machine:

Major Radius, R [m]

Minor Radius, a [m]

Elongation, b/a

Max. $B_{\text {tor }}[\mathrm{T}]$

$\begin{array}{llll}2.6 & 3 & 1.7 & 6 \\ 1.0 & 1.1 & 0.65 & 2.15 \\ 1 & 2 & 2 & 2 \\ 5 & 3.5 & 2 & 5\end{array}$

Qperating:

Max.Current, Io [MA]

2.5

6

2

22

Current Density $\left[\mathrm{A} / \mathrm{cm}^{2}\right]$

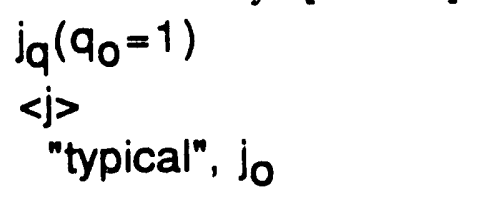

$\begin{array}{rrrr}310 & 190 & 190 & 130 \\ 80 & 80 & 75 & 75 \\ 160 & 120 & 120 & 100\end{array}$

Plasma Density, $\mathrm{n}_{e}\left[\mathrm{~cm}^{-3}\right]$ "typical" $4(13) \quad 5(13) \quad 7(13) \quad 10(13)$

Runaway-Relevant Plasma Parameters During Disruptions:

Stage $\perp\left(T_{\theta}=300 \mathrm{eV}, Z_{e f f}=5\right):$

El. Coll. Frequ. $f_{\theta e}\left[\mathrm{sec}^{-1}\right] \quad 1.4(6) \quad 1.7(6) \quad 2.5(6) \quad 3.5(6)$

$$
f_{\theta O}=Z_{e f f} f_{e \theta}\left[\mathrm{sec}^{-1}\right] \quad 7(6) \quad 8.8(6) \quad 1.2(7) \quad 1.8(7)
$$

El. Field $E_{0}[\mathrm{~V} / \mathrm{cm}] \quad 10(-3) \quad 7(-3) \quad 7(-3) \quad 6(-3)$

Time-to-Relat. tacc [sec] $1.7(-3) \quad 2.4(-3) \quad 2.3(-3) \quad 2.6(-3)$

Stage $11\left(T_{\theta}=10 \mathrm{eV}, Z_{e f f}=3\right)$ :

$\begin{array}{rllll}\text { El. Coll. Frequ. } f_{\theta \Theta}\left[\mathrm{sec}^{-1}\right] & 1.6(8) & 2(8) & 3(8) & 5(8) \\ f_{\Theta O}=Z_{e f f} f_{\theta 0}\left[\mathrm{sec}^{-1}\right] & 5(8) & 6(8) & 9(8) & 1.2(9) \\ \text { El. Field } E_{0}[\mathrm{~V} / \mathrm{cm}] & .8 & .6 & .6 & .55 \\ \text { Time-to-Relat. } t_{a c c}[\mathrm{sec}] & 2.1(-5) & 2.7(-5) & 2.5(-5) & 3.2(-5)\end{array}$ 
TABLE ॥

\section{PARAMETERS}

Pro (see Equ. (11))
TFTR JET DIIID ITER

$7(-4) \quad 4.4(-4) \quad 3.4(-4) \quad 2(-4)$

$\begin{array}{llll}.58 & .72 & 1.0 & 1.4 \\ 17 & 29 & 40 & 70 \\ .017 & .011 & .008 & .005\end{array}$

Stage لll:

Dreicer Field, ED $[\mathrm{V} / \mathrm{cm}]$

Critical energy, $W_{c}[\mathrm{keV}]$

Initial Normalized Field, $\varepsilon_{\mathbf{i}}$
12

.17

.06
15

.27

.038
21

.35

.028
31

.6

.017

TABLE II : Normalized field parameters and required runaway density, Pro, (see Eq. (11)) for various machines as derived from parameters of Table I. 
Iable III: Parameters relevant for runaway generation via trapped hot thermal electrons

\begin{tabular}{|c|c|c|c|c|}
\hline PARAMETERS & TFTR & JET & DIIID & ITER \\
\hline Pra,o & $7(-4)$ & $4.4(-4)$ & $3.4(-4)$ & $2(-4)$ \\
\hline$P_{\text {trap,o }}$ (assumed) & .3 & .3 & .3 & .3 \\
\hline \multicolumn{5}{|l|}{ Normal. Time Limit } \\
\hline$\tau_{f, 0}($ Eq. $(21))$ & 43 & 50 & 52 & 62 \\
\hline \multicolumn{5}{|l|}{$\mathbf{T}_{\text {eo }}=5 \mathrm{keV}$} \\
\hline$t_{\text {trap,th }}[\mu s e c]$ & 13 & 10 & 7.4 & 5 \\
\hline$t_{f, 0} \quad[m s e c]$ & .6 & .5 & .4 & .3 \\
\hline \multicolumn{5}{|l|}{$T_{e o}=10 \mathrm{keV}$} \\
\hline$t_{\text {trap,th }}[\mu s e c]$ & 37 & 28 & 21 & 14 \\
\hline$t_{f, 0} \quad[m s e c]$ & 1.6 & .1 .4 & 1 & .8 \\
\hline \multicolumn{5}{|l|}{$T_{e o}=15 \mathrm{keV}$} \\
\hline$t_{\text {trap,th }} \quad[\mu s e c]$ & 70 & 50 & 40 & 25 \\
\hline $\mathrm{t}_{\mathrm{c}} \quad[\mathrm{msec}]$ & 2.9 & 2.6 & 2.0 & 1.6 \\
\hline
\end{tabular}




\section{Appendix}

\section{Gross Estimate of Potential Energy Gains of Runaways in Disruptions}

In the following, we will summarize the basis for the potential energy gains of "30-60 MeV/MA" used earlier in the paper. In this, we will limit ourselves to a gross estimate of the approximate range of energy gains to be expected in large-tokamak disruptions. The actual energy gains in each case obviously will depend on the drop in discharge current as well as various details of discharge parameters and particle orbits which may vary from one device and discharge to another; although some of the numbers used below are taken from TFTR, it appears that they are not drastically different in other machines - except possibly in a redesigned ITER. Overall, it appears that the drop in discharge current is the main determinant.

For relativistic electrons, the rate of energy gain, $d W / d t=e E c$, is determined by the electric field, $E \sim V_{100 p} / 2 \pi R$, where the overall loop voltage contains three main parts:

$$
V_{\text {loop }} \sim d\left(L_{\text {dis }} I_{\text {tot }}\right) / d t-d\left(\pi R^{2} B_{\text {vert }}\right) / d t-V_{\text {ohm }}
$$

with $L_{\text {dis }}=$ discharge self-inductance, $B_{\text {vert }}=$ vertical magnetic field, and $V_{\text {ohm }}=$ voltage induced by ohmic transformer. Integrating over time, each of the three components will contribute to the overall energy gain

$$
\delta W \sim \delta W_{\text {ind }}+\delta W_{\text {vert }}+\delta W_{o h m}
$$

These contributions will be estimated in the following 
(a) Self-Inductive Eneray Gains. $\delta W_{\text {ind }}$ :

The effective self-inductance seen by a runaway depends on the shape parameters of the discharge and the current density as well as the orbital position of the runaway within the discharge. Assuming a uniform current distribution in a plasma of major radius $R$ and minor radius $a$, we approximately

$$
L \sim\left(\mu_{0} / 2 \pi\right)(2 \pi R)[\ln (8 R / a)-2] \sim\left(\mu_{0} / 2 \pi\right)(2 \pi R) \ln (R / a)
$$

and

$$
\delta W_{\text {ind }} / e \sim\left(\mu_{0} / 2 \pi\right) \ln (R / a) c \delta I_{p}=60 \ln (R / a) \delta I_{\text {tot, } M A}[\mathrm{MeV}]
$$

i.e.

$$
(1 / \theta) \delta W_{f} / \delta I_{p} \sim 60 \ln (\mathrm{R} / \mathrm{a})[\mathrm{MeV} / \mathrm{MA}] \sim 60-80[\mathrm{MeV} / \mathrm{MA}]
$$

for $\mathrm{R} / \mathrm{a} \sim 3-4$. (About $25 \%$ less for b/a $\sim 1.6$ In practice, this basic "inductive potential" normally is reduced somewhat by two effects:

(i) Shielding of the discharge by the conducting vacuum wall: This will depend on the relative current disruption time, $\tau_{\text {dis }}$, vs. the (L/R) time of the wall, $\tau W$, and on relative inductances of wall and discharge. For the general situation with ${ }^{\tau} W^{/} \tau_{\text {dis }} \ll<$, one obtains a fractional reduction of $\delta W_{\text {ind }}$ somewhat smaller than $\tau_{W} / \tau_{\text {dis. }}$. For TFTR, with $\tau_{W} \sim 1 \mathrm{msec}$, and a fast current decay time for $2 \mathrm{MA}$ disruption of about $4 \mathrm{msec}$ this reduction will amount to about $20 \%$. (ii) Partial exclusion/retention of discharge flux from area around major axis (e.g. due to thick copper coils, ohmic transformer etc.): This effect probably normally does not amount to $\leq 10 \%$.

Considering the variations in the various parameters, a range of $\delta \mathrm{W}_{\text {ind }} /\left.\delta\right|_{\text {tot }} \sim+(45-70) \mathrm{MeV}$ appears a reasonable estimate overall.

(b) Eneray Gain due to Vertical Field Changes:

Assuming $R \sim$ const., one obtains

$$
\delta W_{\text {vert }} / \theta \sim(R / 2) \text { c } \delta B_{\text {vert }}
$$

dependent on the actual change during the disruption. Taking the 
initial vertical field

$$
B_{\text {vert,o }}=\left(\mu_{0} / 4 \pi R\right) I_{0}\left\{\ln (8 R / a)+\beta_{p o l}+I_{j} / 2-3 / 2\right\}
$$

Assuming that the disrupting discharge were retained at the original radius, $R$, while $I_{\text {tot }} \rightarrow 0$, with $\beta_{p o l}=0$ and $I_{i} \sim 1 / 2$, we find that the energy of the runaways would be decreased by about

$$
\begin{gathered}
(1 / \theta) \delta W_{\text {vert }} /\left.\delta\right|_{\text {tot }} \sim-c\left(\mu_{o} / 8 \pi\right)\{\ln (\mathrm{R} / \mathrm{a})+.8\} \\
\sim-28[\mathrm{MeV} / \mathrm{MA}]
\end{gathered}
$$

Actually, however, it appears that the equilibrium condition generally is not maintained during the disruption - at least on TFTR. On that device, the percentage change of the vertical field current during a disruption generally is only about $1 / 3-1 / 2$ that of $I_{\text {tot }}$. Correspondingly, it seems more reasonable to approximate

$$
\delta \mathrm{W}_{\text {vert }} / \delta \mathrm{I}_{\text {tot }} \sim-.4 \times 28 \sim-11 \mathrm{MeV} / \mathrm{MA} .
$$

Again, this number has to be further reduced (by about the same percentage as mentioned for $\delta W_{\text {ind }}$ ) due to the shielding by the resistive vacuum wall.

(c) Eneray Loss due to Ohmic Transformer Voltage:

As indicated above, the final runaway energy, of course, also may be reduced by applying a proper negative voltage, $V_{\text {ohm }}$, on the ohmic transformer. The respective reduction is:

$(1 / \theta) \delta W_{o h m}=-(c / 2 \pi R) V_{o h m} \tau_{\text {dis }}$

Unfortunately, this effect generally is not very significant since $V_{\text {ohm }}$ is much smaller than the resistive loop voltage within the disruption. For example, in TFTR, $\mathrm{V}_{\text {ohm }} \leq 100 \mathrm{~V}, \mathrm{R}=2.5 \mathrm{~m}$, and $\tau_{\text {dis }}$ $4 \mathrm{msec}$, so that $\delta W_{\text {ohm }} \sim 8 \mathrm{MeV}$ which can be significant in low-Io discharges, but is not really important in high-Io discharges. 
Overall Eneray Gain $\delta W_{\text {tot }}$ :

As evident from the above discussion, the overall energy gain of runaway electrons will be influenced by the design details of the various machines. In general, an actual overall acceleration potential of about $30-60 \mathrm{MeV} / \mathrm{MA}$ appears to be a fair approximation.

However, we should note again that this does not necessarily mean that the runaways will actually accelerate to an average or even maximum energy given by that value; instead, the final energy value will depend also on other effects, in particular on the confinement of the runaways and their potential energy losses, e.g. due to synchrotron radiation. 


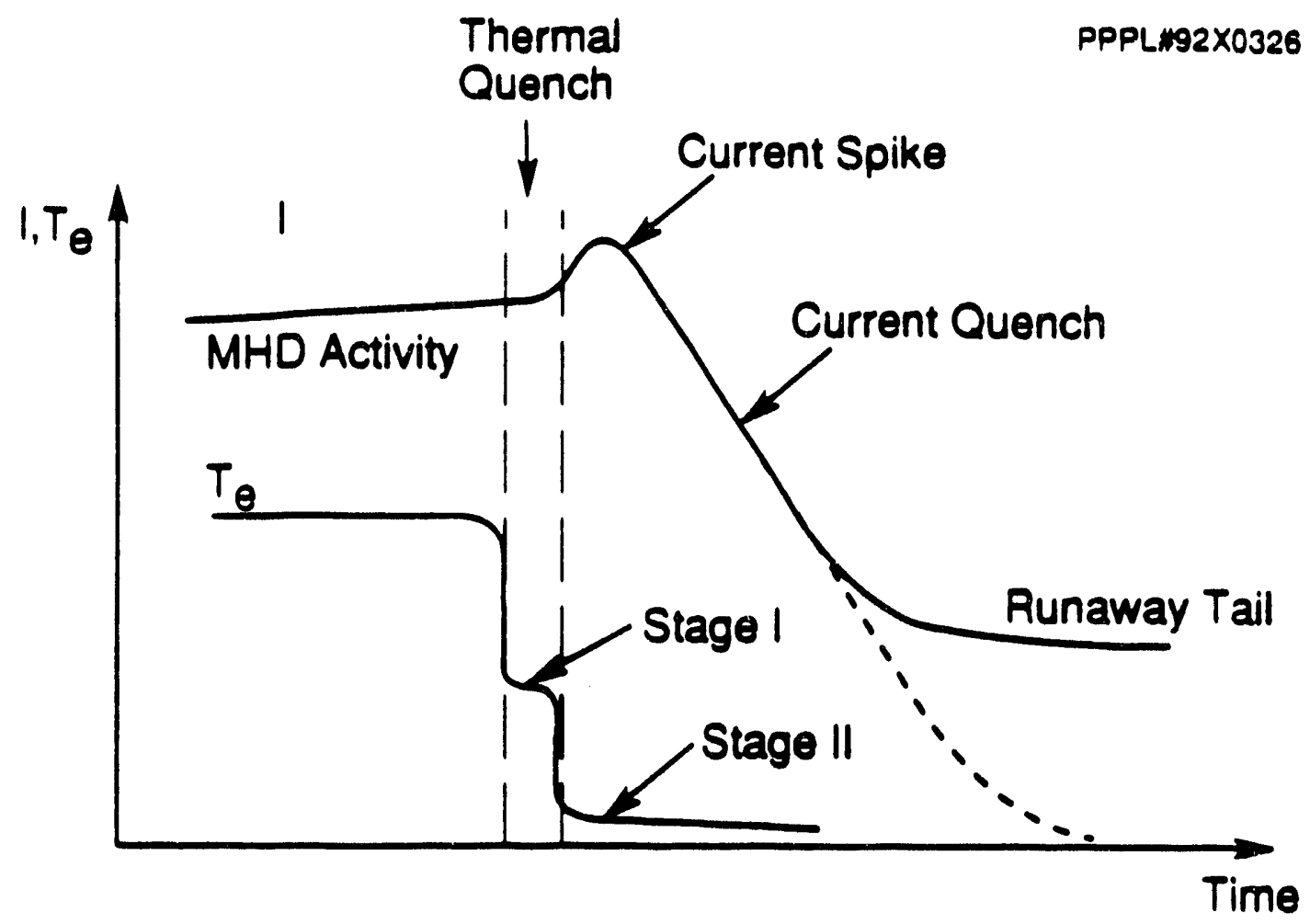

Fig. 1 


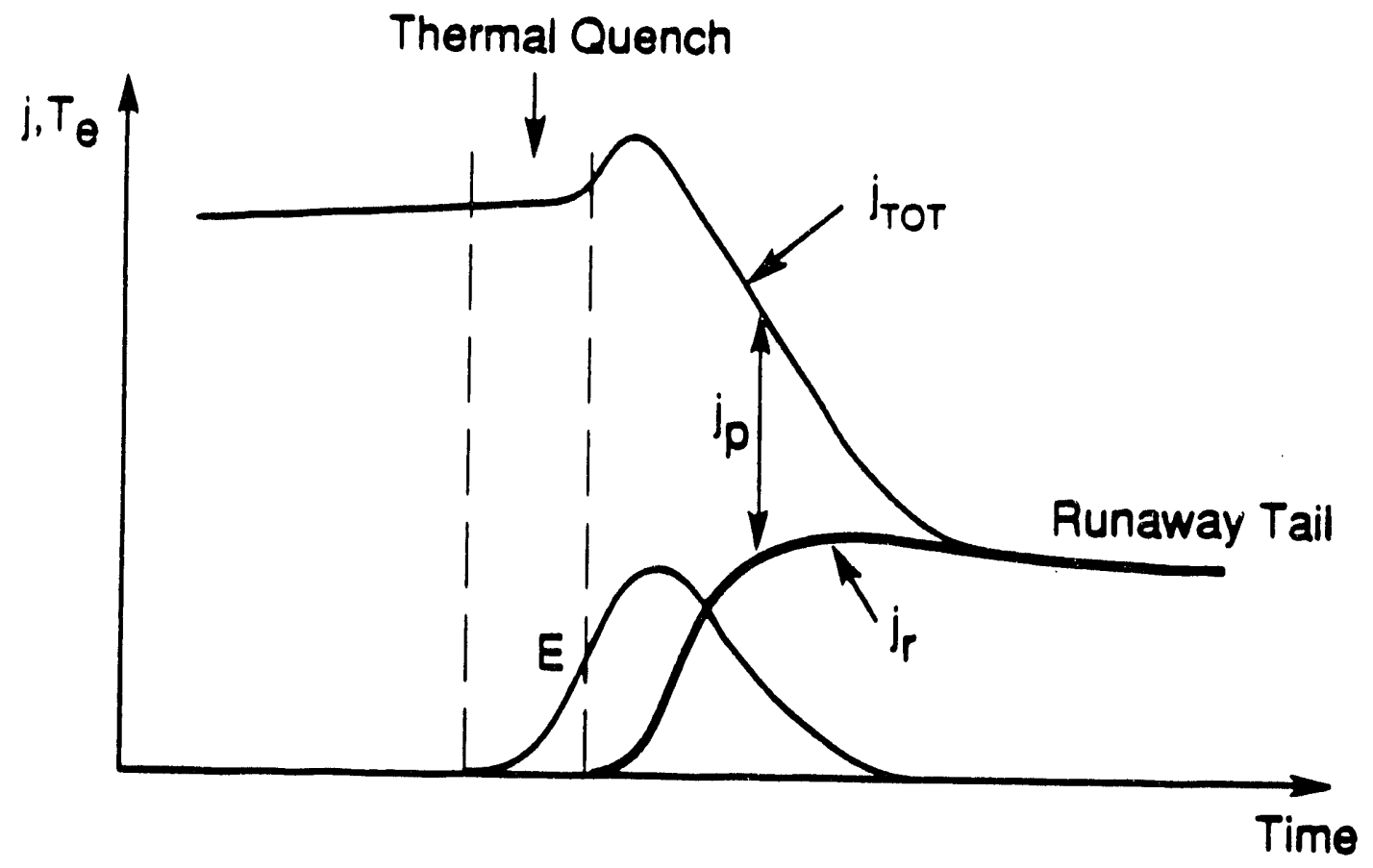

Fig. 2 


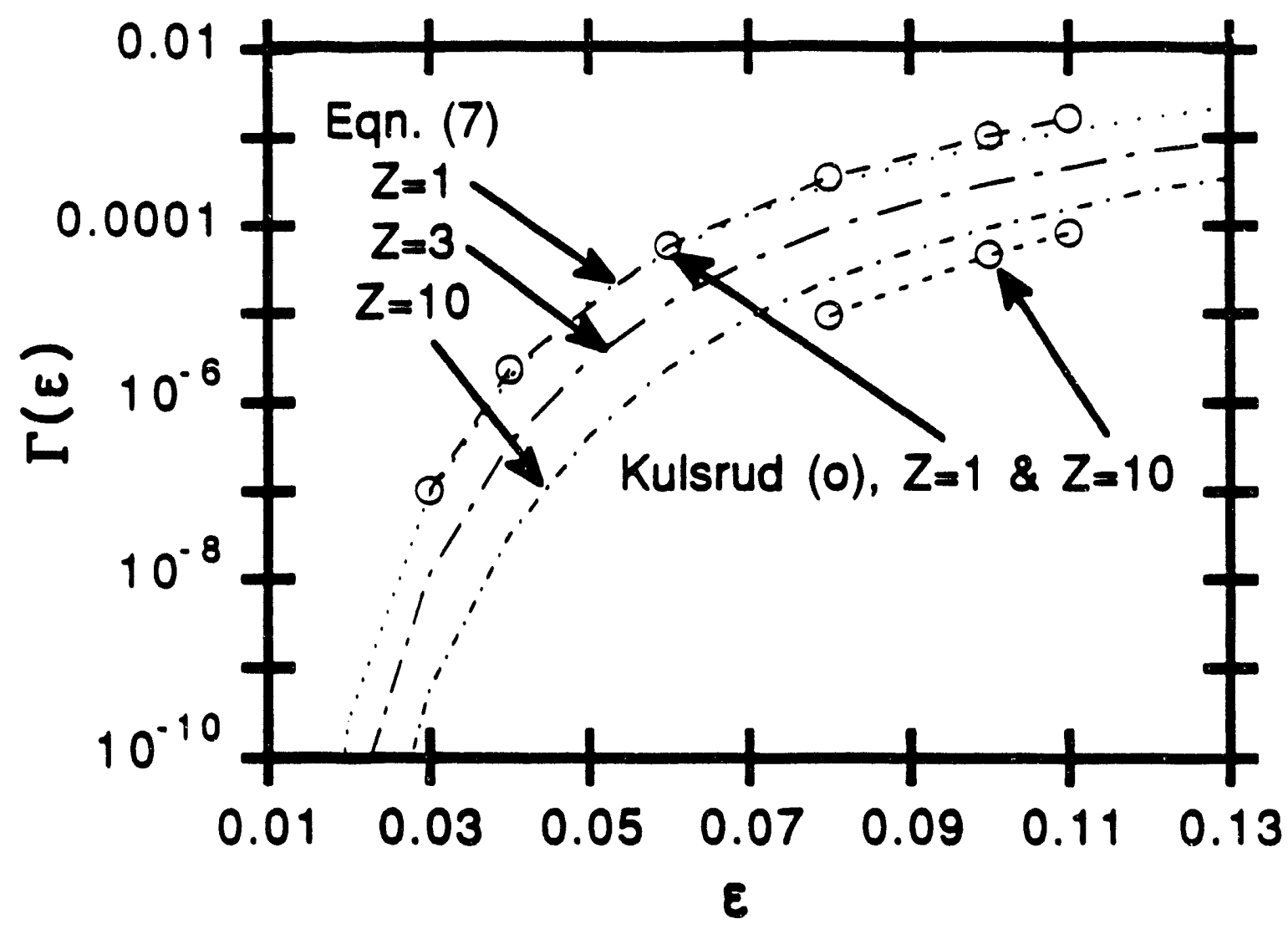

Fig. 3 


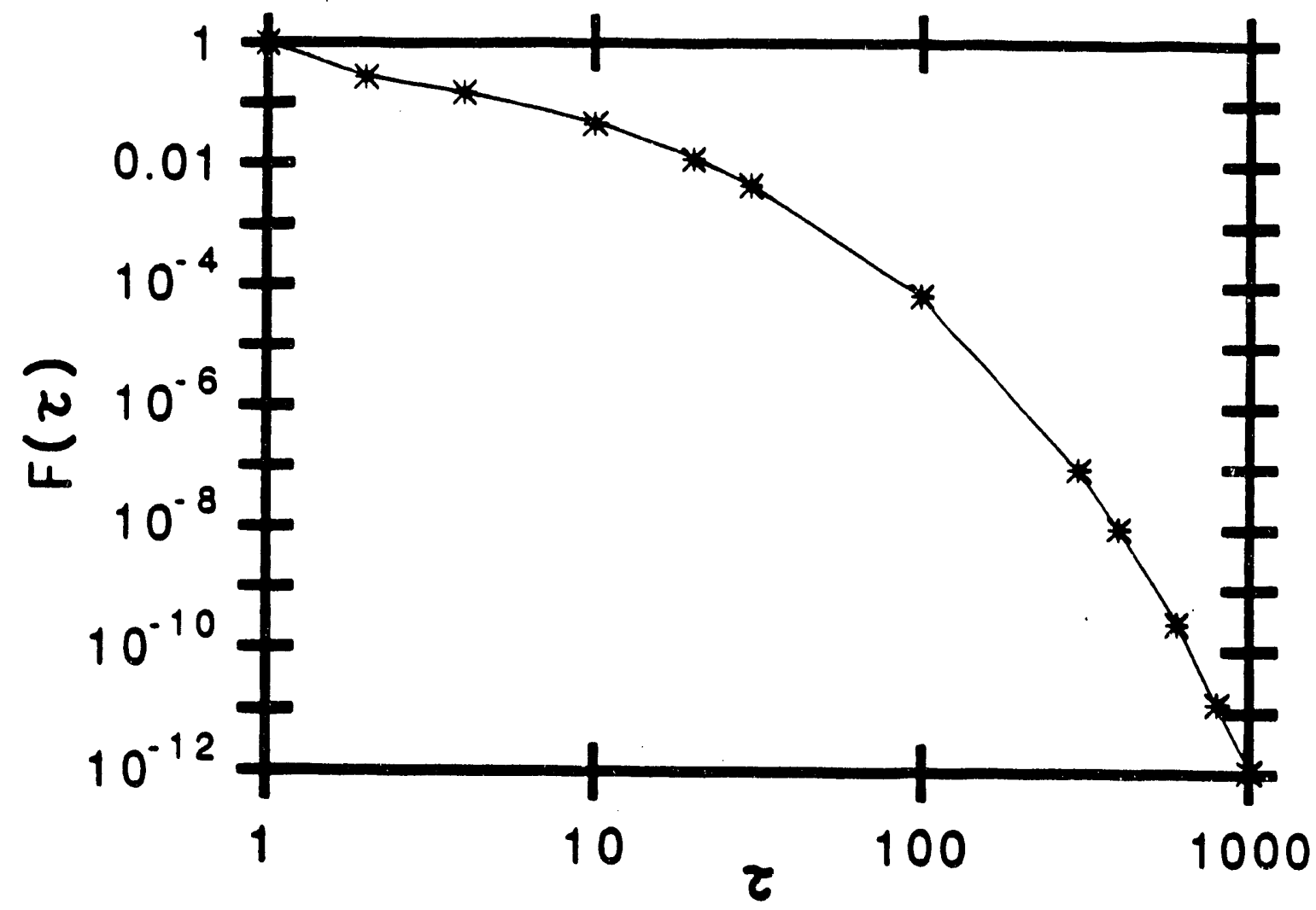

Fig. 4 
Dr. F. Peoloni, Univ. of Wollongong, AUSTRALIA

Prot. M.H. Brennan, Univ. of Sydnoy, AUSTRALIA

Plasme Rosearch Lob., Australlen Nal Univ. AUSTRALIA

Prof. I.R. Jonse, Flinders Unir, AUSTRALIA

Prof. F. Ceap, Inst for Theoretical Physics, AUSTRIA

Prof. M. Hoindier, Instiut for Theoretiscto Physik, AUSTRIA

Prof. M. Gooseans, Astronomiect InctituUt, BELGIUM

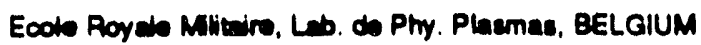

Commisaion-Europeen, OG. XII-Fusion Proo.. BELGIUM

Prof. R. Bouciqut, Rikneurimeritin Gent, BELGIUM

Dr. P.H. Sakenake, Instivio Frica, BRUZIL

Institus Neciond Do Pesquiens Especieir-INPE, BRUZIL

Documents Oriros. Abonic Enoroy of Cenede Lid., CANADA

Or. M.P. Bectynneki, MPB Technologios, Inc., CANADA

Or. H.M. Skamaard, Univ. of Sackalchowen, CANADA

Prof. J. Teictmann, Univ. of Montreal, CANADA

Prot. S.R. Sruentvean, Uriv. Ol Celory, CANADA

Prot. T.W. Johnstion, INRS-Enemgio, CANADA

Dr. R. Botton, Contro cansedien de tusion magndique, CANAOA

Dr. C.R. Jemes, Univ. of Nberta, CANADA

Dr. P. Luktc, Komenaktho Univerezita. CZECHOSLOVAKIA

Tho Librarien, Cutham Leboratory. ENGLAND

Librery, R61, Ruthertord Appleton Laboratory, ENGLAND

Mrs. S.A. Hukchineon, JET Librery, ENGLAND

Dr. S.C. Shermen Univ. of South Pecific, FIJI ISLANDS

P. Mehomen, Univ. of Heleintw, FINLAND

Prot. M.N. Buesece, Ecols Potyruchniquo, , FRANCE

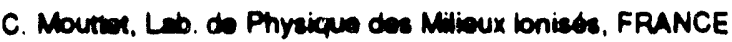

J. Radat, CENCADARACHE - Bat 506, FRANCE

Prot. E. Economow, Univ. of Crew, GREECE

Ms. C. Rinni, Univ. of loannina, GREECE

Dr. T. Muer, Academy Bibliographic Ser., HONG KONG

Preprint Librery, Hungarian Acadorny of SCi.. HUNGARY

Dr. B. DesGupta, Saha lnat of Nuctoer Ptysica, INDIA

Dr. P. Kaw, Inat. for Plasma Roecarch, INDIA

Dr. P. Rocenau, lered inst. of Tectinology, ISRAEL

Librevien, Intemationd Center for Theo Phyeics, ITALY

Miss C. Do Pab, Associezion EURATOMENEA, ITALY

Dr. G. Groseo, Isteuto d Ficica del Plasma. ITALY

Prot. G. Rostangni, lativio Gas lonizzad Ded Cnr, ITALY

Dr. H. Yemeto. Tochibe Rase Dovel Conter, JAPAN
Prof. I. Kawakemi, Hiroshuma Univ., JAPAN

Prof. K. Nishikawa. Hiroshima Univ., LAPAN

Dircetor, Jepen Alomic Enorgy Rosoarch Inst. JAPAN

Prot. S. Itoh, Kyushu Univ., JAPAN

Resorech Into. Ctr., National Instit. for Fusion Science. JAPAN

Prot. S. Tenaka, Kyoto Univ., JAPAN

Librery, Kyolo Univ., JAPAN

Prof. N. Inowe, Univ. of Tokyo, JAPAN

Sacrotary, Pleama Saction, Ebctroluchnical Lab., JAPAN

S. Mori, Technicad Adviecr, LEPI, LAPAN

Dr. O. Minerd, Kumemoto inst of Technolooy. JAPAN

J. Hyeon-Sook, Korea Alomic Energy Poscarch Inet. KOAEA

D.I. Chai. The Korea Adv. Inat. of Sai. \& Tech., KOAEA

Prof. B.S. Liby, Univ. of Weikato, NEW ZEALAND

Inst of Phyeica, Chinese Acad Sa PEOPLE'S REP. OF CHINA

Librey, Inst of Plasma Physics, PEOPLE'S REP. OF CHINA

Tsinghua Univ. Librery, PEOPLE'S REPUBLIC OF CHINA

2. L. S.W. Ina Phyeica, PEOPLE'S REPUBUC OF CHINA

Prol. J.A.C. Cabre, Instituto Superior Tecrico, POATUGAL

Dr. O. Porus, AL I CUZA Univ., ROMANIA

Dr. J. do Villiers, Fusion Studios, AEC. S. AFRICA

Prol. M.A. Hellborg. Univ. of Natal, S. AFPICA

Prof. D.E. Kim, Pohang inst. of Sa. I Tech., SO. KOAEA

Prot. C.I.E.M.A.T, Fusion Division Library, SPAIN

Dr. L. SWmb, Univ. of UMEA, SWEDEN

Librery, Royed inat. of Tectinology. SWEDEN

Prot. H. Winderneon, Chedmers Univ. of Tech., SWEDEN

Centro Phys. Dos Plasmas, Ecole Potylect, SWITZERLAND

Bibliotheak, Inst. Voor Plasma-Fyeich, THE NETHERLANDS

Aset Prot. Dr. S. Cakir, Midde Eeat Tech. Univ., TURKEY

Dr. V.A. Gukthikh, Sai. Roe. Inat. Electrophys.I Apparatus, USSR

Dr. D.D. Ayubv, Siberian Brench of Acadomy of Sai., USSA

Dr. G.A. Eliecer, I.V. Kurchabor Inet, USSA

Librerien, The Uku.SSR Academy of Scionces, USSR

Dr. LM. Kovizhngkh, Inst. of Gonerd Physias, USSR

Komforechungsandepe GmbH, Zentrabibliothek, W. GERMANY

Bibliothok, Inst. FOr Plasmatorechung. W. GERMANY

Prol. K. Schinder, Ruhr-Univereit Bochum, W. GERMANY

Dr. F. Wooner, (ASOEX), Max-Planct-Institul, W. GEAMANY

Librevien, Max-Plenck-Instiur, W. GERMANY

Prol. R.K. Janev, Inat of Phyeice, rUGCSLAVIA 

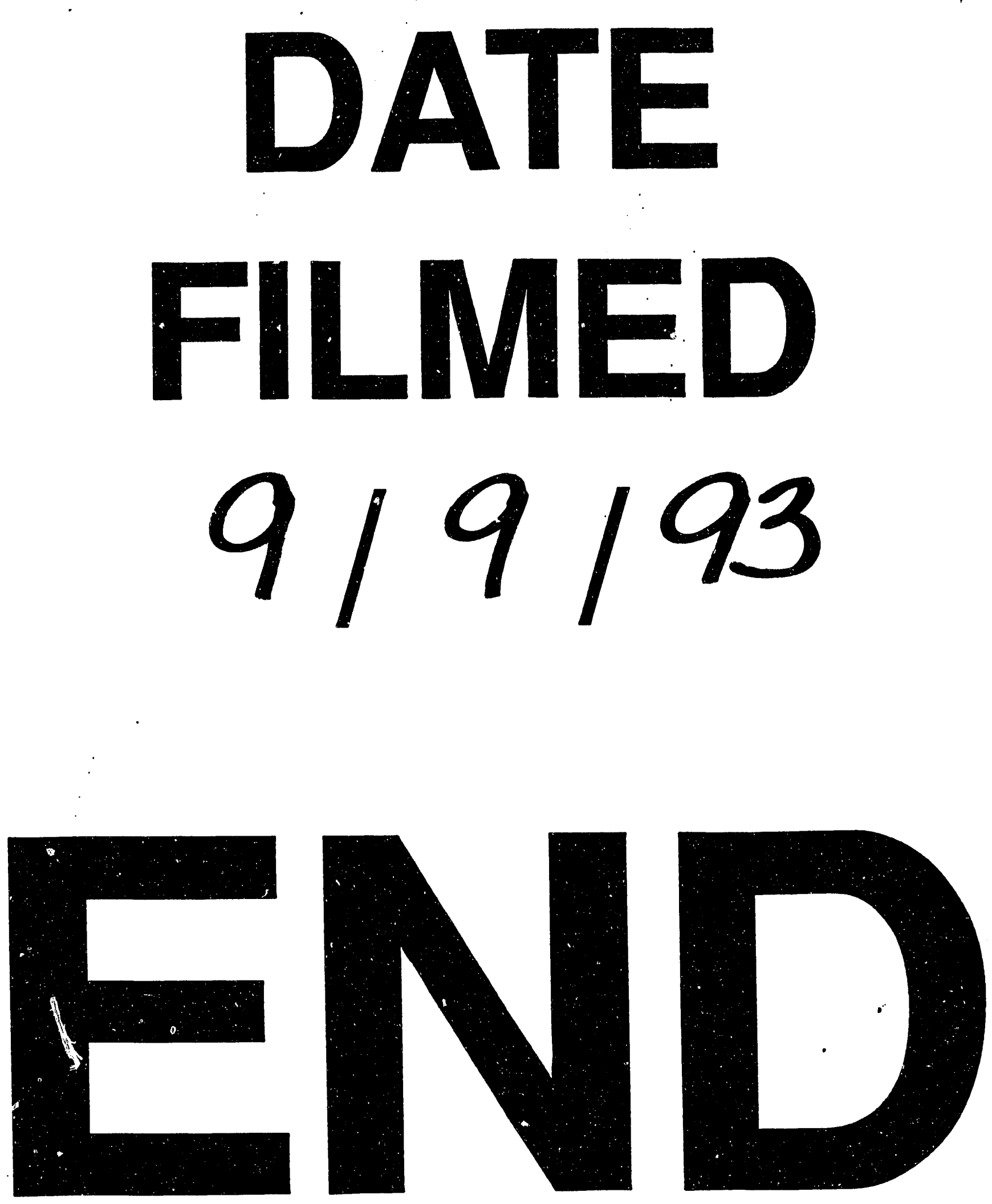
